\title{
OBSERVING CASCADES OF SOLAR BULLETS AT HIGH RESOLUTION. II.
}

\author{
E. SCULlion ${ }^{1,2}$, O. ENGVOLD $^{1}$, Y. LIN $^{1}$, AND L. ROUPPE VAN DER VOORT ${ }^{1}$ \\ ${ }^{1}$ Institute of Theoretical Astrophysics, University of Oslo, P.O. Box 1029, Blindern, NO-0315 Oslo, Norway \\ 2 Trinity College Dublin, College Green, Dublin 2, Ireland; scullie@tcd.ie \\ Received 2015 March 13; accepted 2015 August 3; published 2015 November 25
}

\begin{abstract}
High resolution observations from the Swedish 1-m Solar Telescope revealed bright, discrete, blob-like structures (which we refer to as solar bullets) in the $\mathrm{H} \alpha 656.28 \mathrm{~nm}$ line core that appear to propagate laterally across the solar atmosphere as clusters in active regions (ARs). These small-scale structures appear to be field aligned and many bullets become triggered simultaneously and traverse collectively as a cluster. Here, we conduct a follow-up study on these rapidly evolving structures with coincident observations from the Solar Dynamics Observatory/ Atmospheric Imaging Assembly. With the co-aligned data sets, we reveal (a) an evolving multithermal structure in the bullet cluster ranging from chromospheric to at least transition region temperatures, (b) evidence for cascadelike behavior and corresponding bidirectional motions in bullets within the cluster, which indicate that there is a common source of the initial instability leading to bullet formation, and (c) a direct relationship between coincident bullet velocities observed in $\mathrm{H} \alpha$ and $\mathrm{He}$ II $30.4 \mathrm{~nm}$ and an inverse relationship with respect to bullet intensity in these channels. We find evidence supporting that bullets are typically composed of a cooler, higher density core detectable in $\mathrm{H} \alpha$ with a less dense, hotter, and fainter co-moving outer sheath. Bullets unequivocally demonstrate the finely structured nature of the AR corona. We have no clear evidence for bullets being associated with locally heated (or cooled), fast flowing plasma. Fast MHD pulses (such as solitons) could best describe the dynamic properties of bullets whereas the presence of a multithermal structure is new.
\end{abstract}

Key words: methods: data analysis - methods: observational - Sun: chromosphere - Sun: corona techniques: image processing - techniques: spectroscopic

Supporting material: animations

\section{INTRODUCTION}

A recent study of high spatial, spectral, and temporal resolution observations of the solar chromosphere in $\mathrm{H} \alpha$ $656.28 \mathrm{~nm}$, revealed the existence of small-scale, highly dynamic clusters of bright blob-like features in solar active regions (ARs; Lin et al. 2012, Paper I). In Paper I, linearly propagating blobs observed in emission in the $\mathrm{H} \alpha$ line core characterized the chromospheric component of multiple clusters (containing a total of 28 blobs), which revealed evidence for propagation along (near-horizontal/lateral motion) magnetic threads with velocities in the range of $45-111 \mathrm{~km} \mathrm{~s}^{-1}$. Paper I concluded that the emission and dynamic character of these small-scale features is similar to what one would expect from an MHD fast mode pulse, as a formation mechanism. For discussions of wave-driven mechanisms that might generate such features, we refer to the introduction of Paper I, as well as, Zaqarashvili et al. (2010) and Morton et al. (2011). As deduced in Paper I, observations of any solar phenomenon in only one wavelength channel will reveal limited information on its respective nature and origins. In other words, does the enhanced intensity in $\mathrm{H} \alpha$ line core, characteristic of these features, hint at a more diverse temperature structure to at least TR temperature counterparts of blobs, beyond the clearly observed chromospheric component? Here we conduct a follow-up study of one data set described in Paper I, to investigate the nature of these bright blobs, using combined imaging from EUV channels, via the Solar Dynamic Observatory/Atmospheric Imaging Assembly (SDO/AIA: Lemen et al. 2012) as well as, co-spatial and cotemporal chromospheric diagnostics via the Swedish Solar Telescope (Scharmer et al. 2003) CRisp Imaging Spectro-
Polarimeter (CRISP: Scharmer et al. 2008). Hereafter, we refer to these bright, discretized blob-like structures that appear to propagate laterally across the lower solar atmosphere (rather than outward) as solar "bullets." Their observed bullet-like motion appears to best describe the dynamics and interactions of these features. The bullet analogy will be explored in more detail in the next sections, as well as the causes and consequences of such activity in the context of coronal AR fine-scale structure. The new insights into the nature of solar bullets, offered by AIA, is the motivation behind this follow-up study.

The same AR was imaged in the EUV channels of AIA, which was launched approximately four months prior to the CRISP observations investigated here. The co-spatial and cotemporal time series of the $\mathrm{H} \alpha$ spectral scan sequences (obtained via imaging spectro-polarimetry) can be very precisely compared with hotter EUV channels, as presented herein. Next, we briefly describe the observations, data processing, and co-alignment methods. Following that section, we present the results of our in-depth study into the thermal properties/temperature structure and nature of solar bullets. In the discussion section, we summarize our findings concerning the observed properties of solar bullets across a broad spectral range. Finally, we draw our discussions to a close with a reflection on possible scenarios that could describe the physical formation of solar bullets.

\section{DATA REDUCTIONS}

CRISP is an imaging spectro-polarimeter that includes a dual Fabry-Pérot interferometer (FPI) system similar to that described by Scharmer (2006). For $\mathrm{H} \alpha$, the pre-filter of CRISP 
is $0.49 \mathrm{~nm}$. From 10:58:14-11:30:13 UT on 2010 June 27 th, CRISP was set to cycle continuously through 41 line positions (from $-0.1713 \mathrm{~nm}$ in the $\mathrm{H} \alpha$ blue wing through the line center to $+0.1713 \mathrm{~nm}$ in the red wing, in equidistant steps of $0.0085 \mathrm{~nm}\left(3.9 \mathrm{~km} \mathrm{~s}^{-1}\right)$. The observations targeted the center AR 11084 close to the solar limb. The resulting cadence of the sequential $\mathrm{H} \alpha$ scan sequences is $9 \mathrm{~s}$. By applying the MultiObject Multi-Frame Blind Deconvolution (MOMFBD: van Noort et al. 2005) image restoration technique, the images in the time series are corrected for high frequency atmospheric distortions (beyond the capability of the adaptive optics system correcting in real-time for low order distortions). We used early versions of a CRISP data reduction pipeline (de la Cruz Rodríguez et al. 2015) to prepare the $\mathrm{H} \alpha$ spectral data cubes for this analysis. We explore the fully processed data in the field of view (FOV) with CRISPEX, a versatile code for analysis of multi-dimensional data cubes (Vissers \& Rouppe van der Voort 2012). Together with CRISP observations, space-based $S D O / A I A$ observes the Sun in eight different temperature channels in the (E)UV with an uninterrupted cadence of $12 \mathrm{~s}$. We include in our analysis the following AIA channels: the continuum $170.0 \mathrm{~nm}$ channel (for co-alignment of the images with CRISP), the transition region He II $30.4 \mathrm{~nm}(\log T=4.7)$ line and the AR low coronal lines of Fe Ix $17.1 \mathrm{~nm}(\log T=$ 5.8), Fe XII $19.3 \mathrm{~nm}(\log T=6.2)$ and Fe XIV $21.1 \mathrm{~nm}(\log T=$ 6.3). These are the only AIA wavelengths under consideration because we only want to sample the transition region and nonflaring active coronal lines with detectability in a range up to a few million degrees to compare with bright structures in $\mathrm{H} \alpha$. The standard SolarSoft (SSWIDL) data preparation (aia_prep. pro) is applied to raw AIA images to co-align each AIA channel in the continuum and applies hot pixel, plate scale, and limb fitting corrections. A small spatial offset is expected between the internal SDO/AIA channels on the order of 0.25-0.5 AIA-pixels (Aschwanden \& Boerner 2011). The pixel size of the $\mathrm{H} \alpha$ images is 0 " 06 , which means $\sim 10$ times more resolving power than that of SDO/AIA images ( 0 ". 6 per pixel).

We used the SST turret pointing information to determine the center of the CRISP FOV, which had an accuracy (prior to the 2013 observing season) of $+/-5^{\prime \prime}$ in solar- $x$ and solar- $y$. To achieve sub-AIA pixel accuracy in the co-alignment of AIA with CRISP narrowband images, we cross-correlate photospheric bright points in near simultaneous CRISP wideband images and the AIA continuum channel of $170.0 \mathrm{~nm}$, thereby achieving an accurate co-alignment between CRISP and the transition region and coronal lines of AIA. Photospheric bright points generally exist as discrete, bright magnetic flux concentrations and they are relatively long lived. They are present in abundance in both CRISP $\mathrm{H} \alpha$ near-continuum spectral images and the AIA $170.0 \mathrm{~nm}$ images and so an accurate cross-correlation of the bright points between CRISP and all AIA $170.0 \mathrm{~nm}$ could be achieved in order to co-align the image sequences from both instruments. Since the other AIA channels were previously aligned with $170.0 \mathrm{~nm}$, by default, we achieve a co-alignment with all AIA channels. A similar approach is demonstrated in Scullion et al. (2014). The cadence of the AIA time series differs from CRISP scans by $3 \mathrm{~s}$. In order to be consistent with the $S S T$ cadence, we compare the $S D O$ images in each channel with the closest CRISP time frame. Since the SST is tracking solar rotation in time and the AIA images are now co-aligned and derotate to the first time frame, we only determine the co-alignment correction between

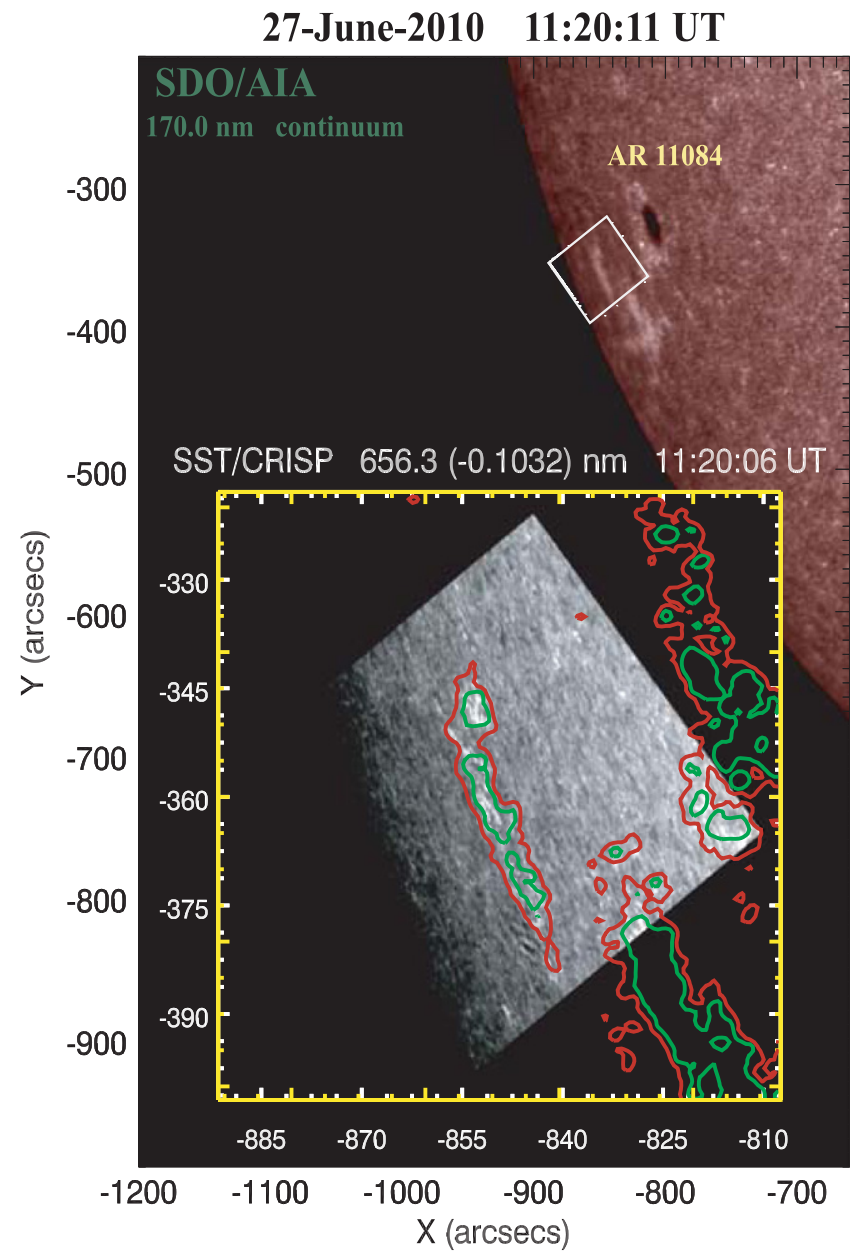

Figure 1. Perspective image of the CRISP FOV with respect to AIA $170.0 \mathrm{~nm}$ is presented. The photospheric $170.0 \mathrm{~nm}$ image is in the background. The CRISP $/ \mathrm{H} \alpha$ FOV is marked as a white box close to the limb sunspot of AR11084. The red and green contours over the $\mathrm{H} \alpha$ gray-scale image (bottom) correspond to photospheric bright points of $170.0 \mathrm{~nm}$, which cross-correlate with coincident bright points in the $\mathrm{H} \alpha$ far red-wing line position $(-0.1032 \mathrm{~nm})$.

CRISP and AIA for the first time frame and apply that correction for the subsequent frames in the observation. Figure 1 presents the pointing of the CRISP FOV (bottom; corrected for solar tilt), with respect to $S D O /$ AIA (background) in the $170.0 \mathrm{~nm}$ channel, after co-alignment and derotation of the images. The red/green contours of the $170.0 \mathrm{~nm}$ photospheric bright points are overlaid onto the $\mathrm{H} \alpha$ continuum image to demonstrate the accurate co-alignment of the observations from both instruments. The accuracy of the co-alignment between CRISP and the AIA channels is at the diffraction limit within $\mathrm{H} \alpha$, as observed with CRISP, i.e., 0.14 arcsec in solar- $x$ and solar- $y$. The resulting FOV of the images is $84^{\prime \prime} \times 84^{\prime \prime}$.

\section{OBSERVATIONS OF A CLUSTER OF SOLAR BULLETS}

In Figure 2, we present the observation FOV in the $\mathrm{H} \alpha$ line core (panel A). On the right side, we present image sequences (top to bottom in time) of two solar bullets (\#1-2) that are coincident in both the $\mathrm{H} \alpha$ line core (upper chromosphere) and He II $30.4 \mathrm{~nm}$ images (transition region). The tracks of these bullets (\#1-2) are over-plotted in panel-A within the FOV. The bullets in $\mathrm{H} \alpha$ have bright cores and faint surroundings and they 

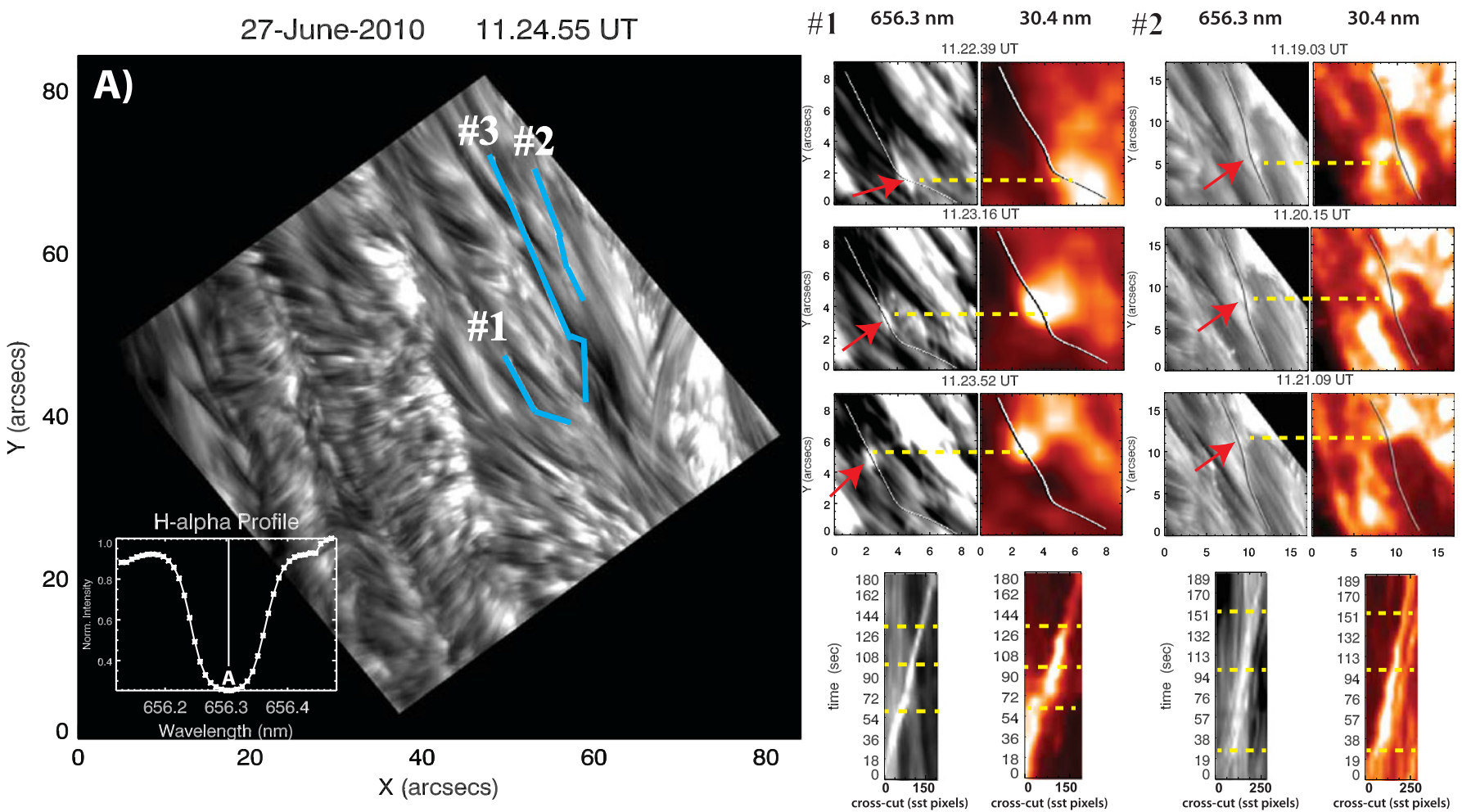

2. CRISP H $\alpha 656.28 \mathrm{~nm}$ line core full FOV is presented in panel A (left) and a number of (blue solid line) bullet tracks (\#1-3) are marked to depict the motion

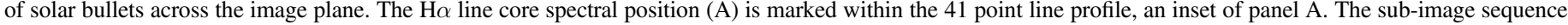

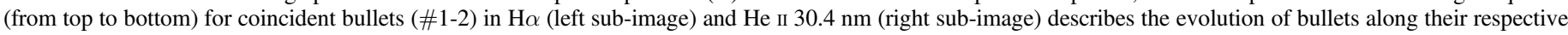

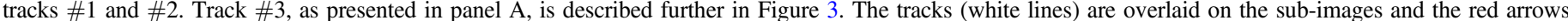

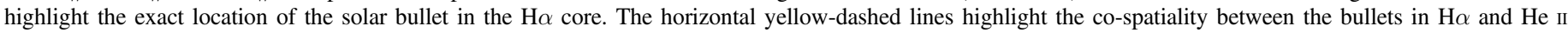

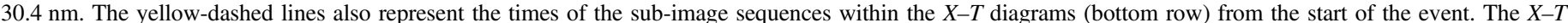

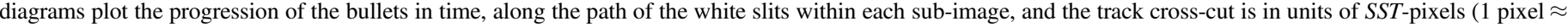

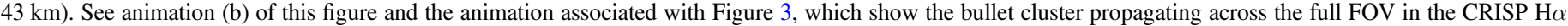

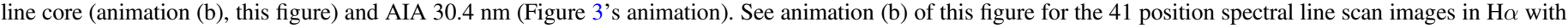
CRISP.

(Animations $\mathrm{a}$ and $\mathrm{b}$ of this figure are available.)

are 0." $3-0$ !" $4(\sim 300 \mathrm{~km})$ in diameter. Both $\mathrm{H} \alpha$ bright core intensities and $30.4 \mathrm{~nm}$ bullet intensities vary by $10 \%-20 \%$ across the cluster.

A realistic comparison of bullet brightness in $\mathrm{H} \alpha$ and $30.4 \mathrm{~nm}$ should take into account the large difference in background brightness at these two wavelengths. Typically, $30.4 \mathrm{~nm}$ bullets appear brighter against their background, with bullet intensities 2.2-2.8 times greater than background. Whereas, $\mathrm{H} \alpha$ bullets are relatively less bright because they have intensities only 1.3-1.5 times greater than background intensity. The bullets in $\mathrm{H} \alpha$ and $30.4 \mathrm{~nm}$ are spatially and temporally matched in cases \#1 and \#2 with a dashed yellow line. The distance-time $(X-T)$ diagrams, shown in Figure 2 (right side, bottom row), corresponds to the motion of the bullets with constant velocity for a lifetime of $180 \mathrm{~s}$, after they first appear in both channels. From the $X-T$ diagrams, we find that the velocities of the $\mathrm{H} \alpha$ bullets in the plane of the sky exist in the same velocity range, as reported in Paper I. Importantly, there is an associated $30.4 \mathrm{~nm}$ counterpart (for the full duration in bullet \#1, but intermittently in bullet \#2) that is a notable and important difference in their morphology. In the case of bullet $\# 2$, the $\mathrm{H} \alpha$ and $30.4 \mathrm{~nm} X-T$ diagrams reveal that the bullets do not always appear and disappear (from their respective spectral channel) at exactly the same time.

In Figure 3, we present similar image sequences (evolving in time from panel A-D) for a partially coincident $\mathrm{H} \alpha$ and
$30.4 \mathrm{~nm}$ bullet that represents track \#3 (see Figure 2-panel A). By partially coincident, we mean that the bullet is only coincident in $\mathrm{H} \alpha$ and $30.4 \mathrm{~nm}$ in panel B (matched with a yellow dashed line, also represented in time in panel E). The bullet itself is made identifiable by blue boxes in each subimage. The spectral evolution in this case is more complicated, such that the bullet initially appears in $\mathrm{H} \alpha$ propagating along the white bullet track, as shown in panel A. The $30.4 \mathrm{~nm}$ counterpart of the $\mathrm{H} \alpha$ bullet first appears after $t=72 \mathrm{~s}$. The coincident bullet in $30.4 \mathrm{~nm}$ first appears in panel B and increases in intensity along the white track until $t=202 \mathrm{~s}$ (see panel E). At the same time, a bright bullet in $\mathrm{H} \alpha$ continues to propagate along the white track beyond $t=202 \mathrm{~s}$. In this case, the bullet's thermal structure appears to change multiple times as it evolves along the track and the onset of the $30.4 \mathrm{~nm}$ component appears coincident with a sharp change in direction of the $\mathrm{H} \alpha$ bullet (i.e., within the white-dashed box in panel C). The consecutive nature of hot and cool bullets, and their association with a potential "trigger" event as inferred with the sharp change of direction in the white track, reveals a new characteristic in describing the nature of bullets.

\subsection{Coincident He II $30.4 \mathrm{~nm}$ and $\mathrm{H \alpha}$ Bullets}

Despite the large difference in the spatial resolution of the CRISP $\mathrm{H} \alpha$ and AIA $30.4 \mathrm{~nm}$, a comparison of the co-temporal and co-spatial time series reveal multiple examples of 
11.18.27 UT

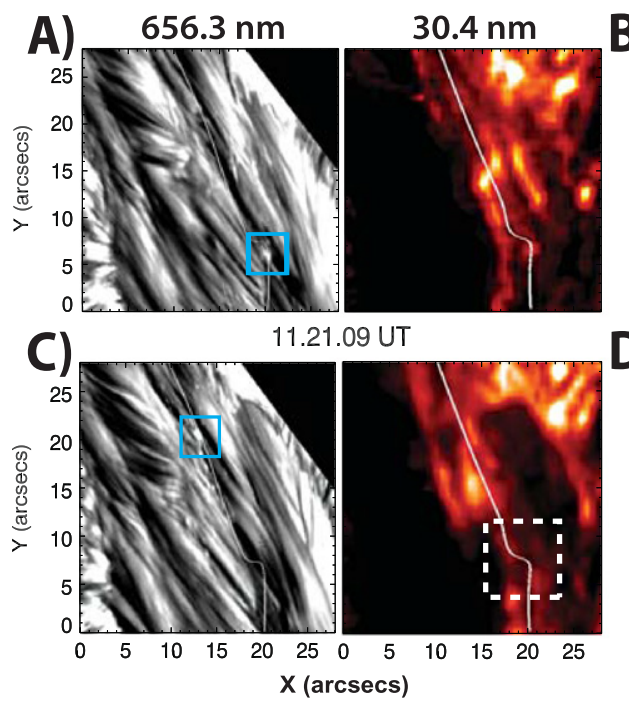

11.19.30 UT

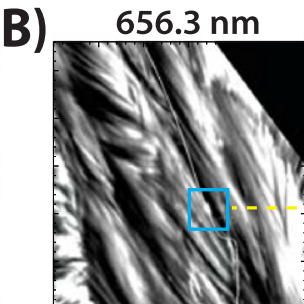

D)

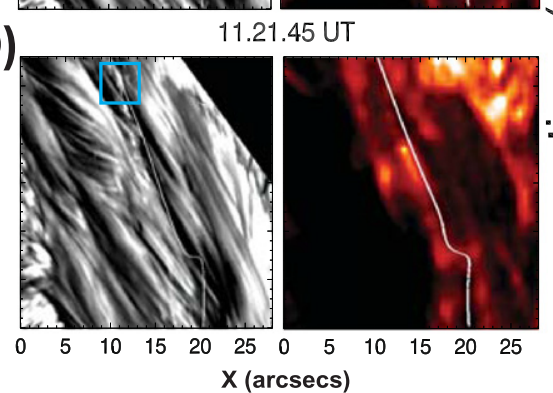

E)

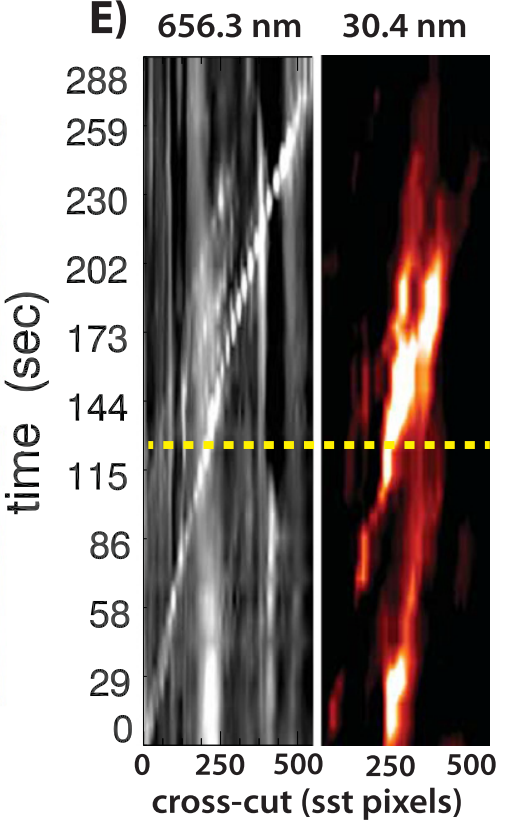

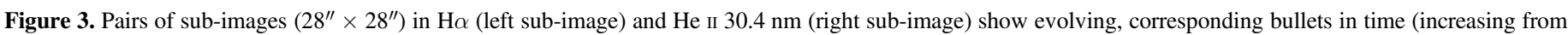

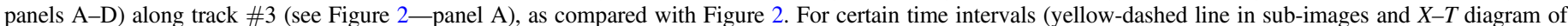

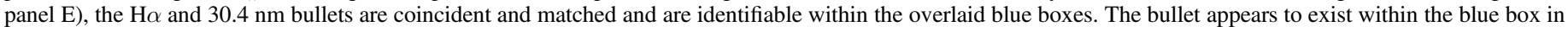
all time frames but only for part of that interval in $30.4 \mathrm{~nm}$. The white dashed box identifies a notable deviation in the trajectory of the bullet motion.

(An animation of this figure is available.)

coincident high speed, small-scale bullets in both channels and with a common apparent lifetime in both channels. Many $\mathrm{H} \alpha$ bullets appear to have fairly strong intensity contrasts with the background signal and they are generally rounded, but some are elongated. The $30.4 \mathrm{~nm}$ bullets appear larger and possibly more diffuse, i.e., the boundary of the bullet feature is less distinct. In total, there are 13 instances of common $30.4 \mathrm{~nm}$ and $\mathrm{H} \alpha$ bullets. Table 1 presents the apparent velocity and intensities of these bullets in these channels, comparatively. Eleven examples of these are presented in Figure 4 (top). In the 11 panels, we detect 6 examples of one-time coincident bullet formation in $\mathrm{H} \alpha$ together with $30.4 \mathrm{~nm}$ (i.e., diagrams \#5, \#7, $\# 8$, \#10, \#12, and \#14). In the remaining 5 bullets (\#4, \#6, $\# 9$, \#11, and \#13), we detect repetitions of coincident $\mathrm{H} \alpha$ and $30.4 \mathrm{~nm}$ bullet formation (most notably in diagram \#1) along the same track and with variable time intervals between their respective formation. We refer such repetitive activity, associated with bullets along the same path, as a thread of bullets. Coincident bullets are such that the bullet tracks are overlapping or very much adjacent to each other during their lifetime and they must follow the same trajectory for some period of time, such as those displayed in Figure 4. The most extreme example, with respect to the lifetime of coincident bullets observed in $\mathrm{H} \alpha$ and $30.4 \mathrm{~nm}$, was where a $\mathrm{H} \alpha$ bullet had a lifetime 5.5 times shorter than its $30.4 \mathrm{~nm}$ counterpart. A sub-plot [1] within the histogram panel in Figure 5, demonstrates that there a very linear relationship between these 13 coincident bullets, with respect to their velocity correspondence (outlined in Table 1). However, there is an inverse relationship when considering their respective intensity variations (sub-plot [2]). There is clearly a strong statistical relationship between many bullets formed in $\mathrm{H} \alpha$ and those appearing in $30.4 \mathrm{~nm}$. This implies that there is a strong connecting physical process present to explain the dynamic, internal thermal changes in the bullets.
Table 1

Several High Speed Bullets Are Selected from $\mathrm{H} \alpha$ and $30.4 \mathrm{~nm}$

\begin{tabular}{|c|c|c|c|}
\hline $\begin{array}{l}\text { Vel. } \mathrm{H} \alpha \\
\left(\mathrm{km} \mathrm{s}^{-1}\right)\end{array}$ & $\begin{array}{l}\text { Vel. He II } 30.4 \mathrm{~nm} \\
\left(\mathrm{~km} \mathrm{~s}^{-1}\right)\end{array}$ & Intensity $\mathrm{H} \alpha$ & Intensity $\mathrm{He}$ II $30.4 \mathrm{~nm}$ \\
\hline $54 \pm 9$ & $62 \pm 2$ & $3300 \pm 130$ & $410 \pm 20$ \\
\hline $66 \pm 3$ & $\ldots$ & $3280 \pm 170$ & $\ldots$ \\
\hline $44 \pm 5$ & $\ldots$ & $3280 \pm 80$ & $\ldots$ \\
\hline $52 \pm 4$ & $62 \pm 8$ & $3350 \pm 70$ & $480 \pm 60$ \\
\hline$\ldots$ & $65 \pm 2$ & $\ldots$ & $390 \pm 10$ \\
\hline$\ldots$ & $60 \pm 3$ & $\ldots$ & $380 \pm 20$ \\
\hline $45 \pm 14$ & $\ldots$ & $2810 \pm 30$ & $\ldots$ \\
\hline $73 \pm 1$ & $85 \pm 2$ & $3680 \pm 160$ & $280 \pm 20$ \\
\hline $89 \pm 6$ & $88 \pm 3$ & $3650 \pm 100$ & $320 \pm 20$ \\
\hline $89 \pm 20$ & $82 \pm 2$ & $3480 \pm 80$ & $270 \pm 10$ \\
\hline $47 \pm 1$ & $53 \pm 1$ & $3170 \pm 90$ & $380 \pm 100$ \\
\hline $81 \pm 1$ & $85 \pm 24$ & $3640 \pm 170$ & $320 \pm 20$ \\
\hline $62 \pm 7$ & $64 \pm 13$ & $3050 \pm 130$ & $530 \pm 20$ \\
\hline $95 \pm 6$ & $\ldots$ & $3170 \pm 100$ & $\ldots$ \\
\hline$\ldots$ & $68 \pm 2$ & $\ldots$ & $410 \pm 20$ \\
\hline $72 \pm 17$ & $68 \pm 11$ & $3530 \pm 120$ & $410 \pm 47$ \\
\hline $72 \pm 3$ & $79 \pm 2$ & $3490 \pm 70$ & $480 \pm 20$ \\
\hline $95 \pm 36$ & $99 \pm 2$ & $3390 \pm 40$ & $490 \pm 60$ \\
\hline $77 \pm 1$ & $82 \pm 13$ & $3410 \pm 140$ & $610 \pm 140$ \\
\hline $97 \pm 16$ & $107 \pm 15$ & $3440 \pm 130$ & $400 \pm 50$ \\
\hline$\ldots$ & $89 \pm 11$ & $\ldots$ & $430 \pm 40$ \\
\hline
\end{tabular}

Note. Here, 13 bullets are coincident in both wavelengths and 4 bullets are detected only in $\mathrm{H} \alpha$ and 4 only in $30.4 \mathrm{~nm}$. The velocities of these bullets in the plane of the sky are in units of $\mathrm{km} \mathrm{s}^{-1}$ and their mean intensities are in arbitrary units.

\subsection{Statistical Analysis of the Bullet Cluster}

In order to investigate the thermal structuring and physical characteristics of bullets further, we carried out a statistical analysis of all detectable bullets in the FOV for the full 

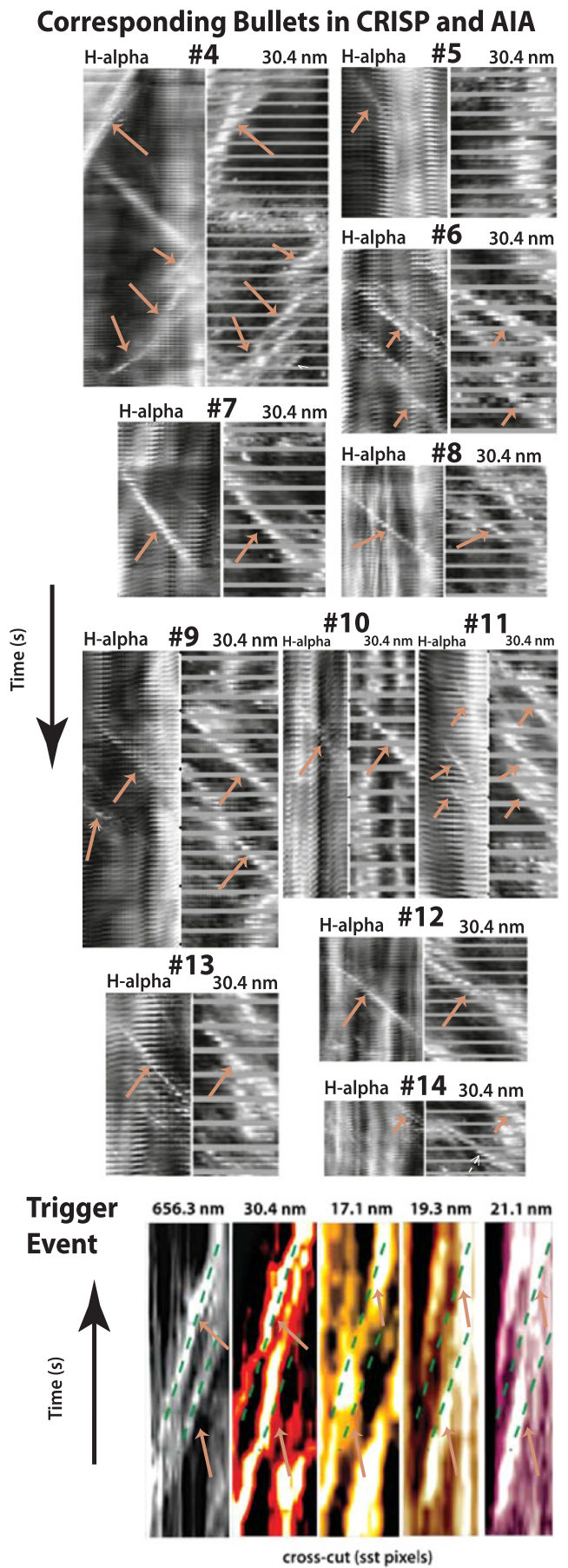

Figure 4. Top: grayscale distance-time $(X-T)$ diagrams for corresponding and coincident $\mathrm{H} \alpha$ and $30.4 \mathrm{~nm}$ bullets (\#4-14) from bullet tracks. The track data is extracted from splines on the co-aligned CRISP and AIA FOV and individual bullets are marked with orange arrows. Time is positive in the downward direction along the $y$ axis. Hence, as time advances forward the bullets evolve along the slit cross-cut (SST pixels) along the $x$ axis, from left to right, in all panels. Sequential horizontal bands in $30.4 \mathrm{~nm}$ are time intervals due to the effect of different cadence in the instruments. Bottom: color-scale $X-T$ diagrams for the trigger event presented in Figure 9, which reveals sequences of relatively hot bullets, emitting across a broad range of temperatures. Time is positive in the upward direction along the $y$ axis. The multithermal properties emanating from a source region are largely concentrated within the boundaries of the green dashed lines.

observation in $\mathrm{H} \alpha$, as well as in the hotter channels of AIA. The selection criteria for what one can describe as a bullet is constrained by the intensity of the bullet relative to the background (deduced from $X-T$ diagrams) and the size of the feature moving across the plane of the sky in the time series. The condition for selection/inclusion of a bullet into the data set, was based on the intensity ratio of the bullet to the background level in each wavelength channel (set to at least $15 \%$ above a background level, determined from the $X-T$ data array), as well as, the presence of a propagation along the length of the track, as measured from the bullet track $X-T$ diagrams (for e.g., see Figure 4). In other words, all bullets have a detectible line of propagation within the $X-T$ diagrams. For the observation duration of $1919 \mathrm{~s}$, we detected a total of 78 individual bullet tracks in $\mathrm{H} \alpha, 43$ tracks in $30.4 \mathrm{~nm}, 15$ tracks in $17.1 \mathrm{~nm}, 20$ tracks in $19.3 \mathrm{~nm}$, and 15 tracks in $21.1 \mathrm{~nm}$.

The results of this statistical approach are presented in Figure 6. From the first interpretation of this compilation of bullet tracks, we find that not all of the bright bullets in $\mathrm{H} \alpha$ (black tracks in panel A) have a coincident signature in the hotter channels (colored tracks). From panel A, it is immediately apparent that the number density of detected bullets is significantly greater in the cool channels (below $100,000 \mathrm{~K}$ ) compared with hotter channels. The $30.4 \mathrm{~nm}$ channel has the closest formation temperature to $\mathrm{H} \alpha$ and, as a result, has the closest correspondence with the detected $\mathrm{H} \alpha$ bullets. There are significantly fewer detections of bullet activity in the hotter channels $(>100,000 \mathrm{~K})$. The vast majority of detected bullets propagate from bottom-right to top-middle within the FOV. They propagate roughly parallel to the solar limb as a cluster.

Histograms of the measured velocities, lifetimes and track lengths of all bullets in all spectral channels, are presented in panels D-F. A Gaussian profile has been fitted to the $\mathrm{H} \alpha$ distribution for panels D and E. The bullet velocity distribution in the cluster peaks at $52 \mathrm{~km} \mathrm{~s}^{-1}$ with an FWHM of $+/-33 \mathrm{~km} \mathrm{~s}^{-1}$. The tail of the velocity distribution extends out to a maximum of $145 \mathrm{~km} \mathrm{~s}^{-1}$, i.e., corresponding to the fastest propagating $\mathrm{H} \alpha$ bullet. This outer limit is marked with a vertical dashed line in panel A and labelled $b$. There exists a set of bullets outside of the fitted distribution with higher velocities beyond $b$, which features only bullets from the hottest AIA channels, i.e., 19.3 and $21.1 \mathrm{~nm}$. The fastest bullet detected was in the coronal $19.3 \mathrm{~nm}$ channel with an apparent velocity of $200 \mathrm{~km} \mathrm{~s}^{-1}$. The significance of these bullets will be explored in the cascade trigger events sub-section.

Statistical analysis of the bullet lifetimes within the main cluster, is presented in Figure 6 panel E. A fitted Gaussian profile has shown that most $\mathrm{H} \alpha$ bullets survive for $75 \mathrm{~s}$ with HWHM of lifetimes of $+135 \mathrm{~s}$. The hottest (19.3 and $21.1 \mathrm{~nm})$ detected bullet lifetimes are also most commonly occurring in the 35-95 s time interval (with peaks at $65 \mathrm{~s}$ in $21.1 \mathrm{~nm}$ and $70 \mathrm{~s}$ in $19.3 \mathrm{~nm}$ ), whereas, the relatively cooler 17.1 and $30.4 \mathrm{~nm}$ bullets have a peak lifetime in the 155-215 s time interval. Another notable feature of the total cluster of tracks (panel A) in the FOV is the preferential/predominant direction of motion (indicated by the large black arrow). We can reveal from the velocity distribution map (panel B and C) that there are two distinct threads/flows within the main cluster of bullets, i.e., a relatively high velocity, long length thread (light blue tracks) on the left side of the cluster, and a low velocity, short length thread (dark blue) on the right side of the cluster. This indicates that most bullets propagate with a constant velocity throughout the observation. This is not surprising since the $X-T$ diagrams of all tracks describe bullet profiles, which is 


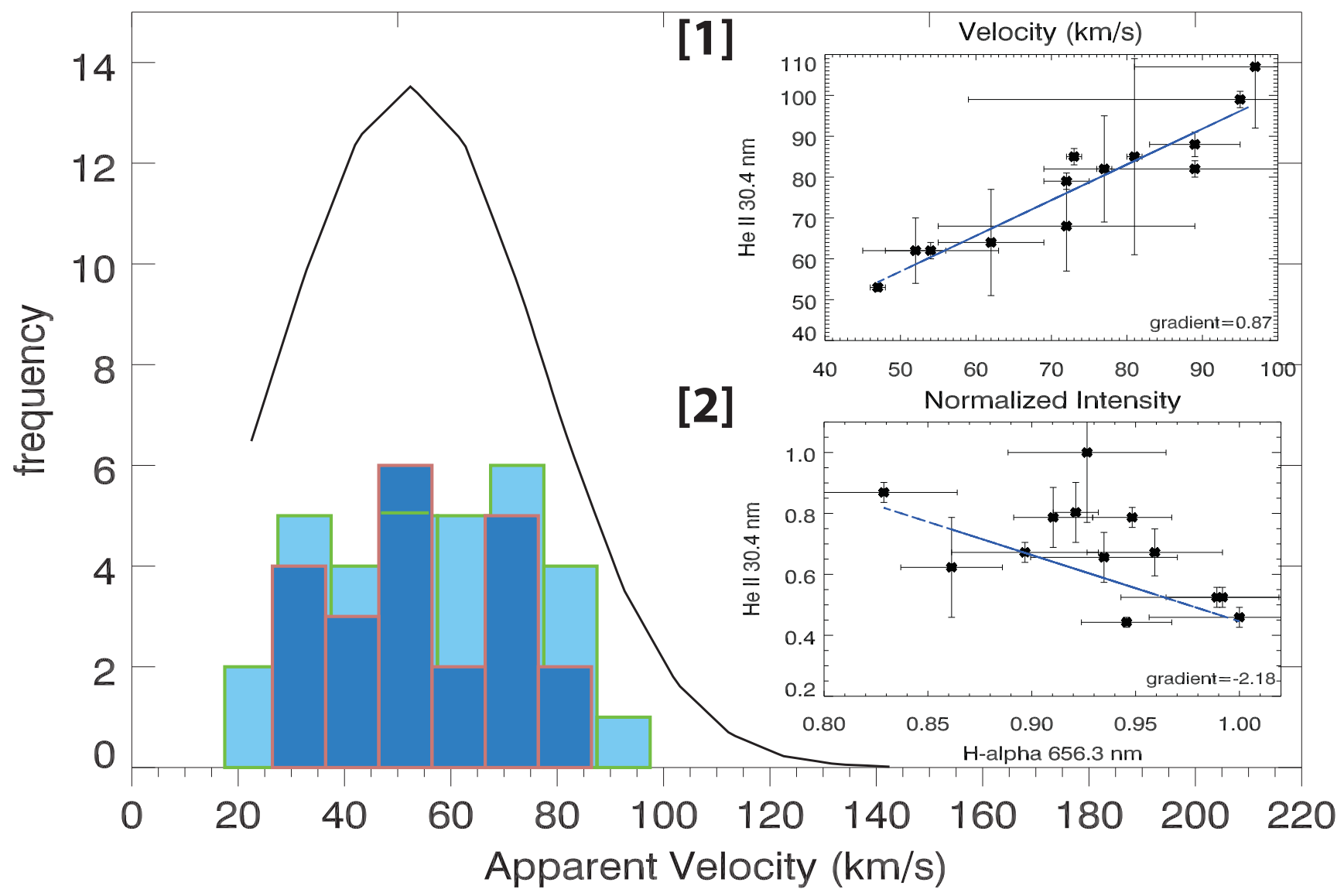

Figure 5. Examples of coincident $\mathrm{H} \alpha$ bullet tracks (black) and $30.4 \mathrm{~nm}$ bullet tracks (red). The velocity distributions of these $\mathrm{H} \alpha$ bullets (light blue) and $30.4 \mathrm{~nm}$ bullets (dark blue) are presented in the histogram (top right). The sub-plot [1] within the histogram represents the linear relationship (fitted dashed line) between coincident bullets with respect to bullet velocity. Sub-plot [2] describes an inverse relationship between respective $\mathrm{H} \alpha$ and $30.4 \mathrm{~nm}$ coincident bullets with line fits over plotted.

suggestive of propagation with constant velocity. As a result, a larger bullet velocity with a similar lifetime will result in a greater track length. The bullet with the largest lifetime and length appeared in $30.4 \mathrm{~nm}$ with values of $725 \mathrm{~s}$ and $27.5 \mathrm{Mm}$, respectively. The shortest track length bullet is $2.7 \mathrm{Mm}(=3 ! .7)$ and exists within the $17.1 \mathrm{~nm}$ channel. The peak in the distribution (1) for $\mathrm{H} \alpha$ bullet track lengths occurs at a length of $6.2 \mathrm{Mm}$, which is in close agreement with 17.1, 21.1, and $19.3 \mathrm{~nm}$ distributions, which peak at the $8-1 \mathrm{Mm}$ range. However, a second Gaussian distribution (2) was fitted to the $30.4 \mathrm{~nm}$ track length distribution, which appears to constitute the tail end of (1). The peak of distribution (2) occurs at $12.3 \mathrm{Mm}$ for $30.4 \mathrm{~nm}$. It may be that there is a bi-modal distribution in the lengths of $\mathrm{H} \alpha$ bullets since there is indeed a fairly large density of bullets at $11.8 \mathrm{Mm}$, which is in close agreement with the peak of distribution (2). The average track length, considering the mean of both distributions (1) and (2), is $9.3 \mathrm{Mm}$ with a FWHM of $+/-7.3 \mathrm{Mm}$. There appears to be a stronger correlation between bullets in $\mathrm{H} \alpha$ (light blue bars in the histogram) and $30.4 \mathrm{~nm}$ (dark blue bars in the histogram) with respect to apparent velocity, but less so with respect to lifetime and track length distributions.

In the next section, we will discuss the evolution of a number of bullet cascades which could be identified within the main cluster. Within the main cluster, we can identify three distinct cascades of bullets, namely cascades A, B, and C. Cascade C is composed of the initial high speed, long length bullets of the main cluster (i.e., left-side of Figure 6 panels B and C). Cascade A is composed of the right-side (relatively slower and short length) bullets that appear later in time and further along toward to top half of the FOV. Likewise, cascade B occurs later in the evolution and is relatively small-scale, compact cascade formed within the top half of the cluster.

\subsection{Cascades $A$ and $B$}

In Figure 7, we present a sequence of images (left to right) describing evolving bullet tracks for cascades A (top row) and B (bottom row). Cascade A has a limited thermal structure (only bullets for $\mathrm{H} \alpha$ and $30.4 \mathrm{~nm}$ are present) compared with cascade B, which contains bullets from 17.1, 21.1, and $193 \mathrm{~nm}$. For both cascades, all bullets propagate diagonally upward and tend to fan outward (avalanche effect) from the boxed source regions, labelled source $\mathrm{A}$ and source $\mathrm{B}$.

Cascade A evolves over an interval of $585 \mathrm{~s}$ and the formation of two near-parallel threads (\#1 and \#2, see 11:10:10 UT panel) clearly generate from source A. The cascade starts in source A as 3 bullets, $2 \mathrm{H} \alpha$ and $130.4 \mathrm{~nm}$ bullet, at 11:00:25 UT $\left(t_{0}\right)$. At this time, the $30.4 \mathrm{~nm}$ bullet appears to be coincident with one $\mathrm{H} \alpha$ bullet constituting the start of cascade thread \#1. The third bullet in $\mathrm{H} \alpha$ constitutes the onset of cascade thread \#2. Within 3 minutes the $\mathrm{H} \alpha$ bullet in thread \#2 can be seen to evolve into a $30.4 \mathrm{~nm}$ bullet, i.e., the black track connects with the start of a $30.4 \mathrm{~nm}$ red track (Figure 7, top row of panel 2). In the same time interval, the 

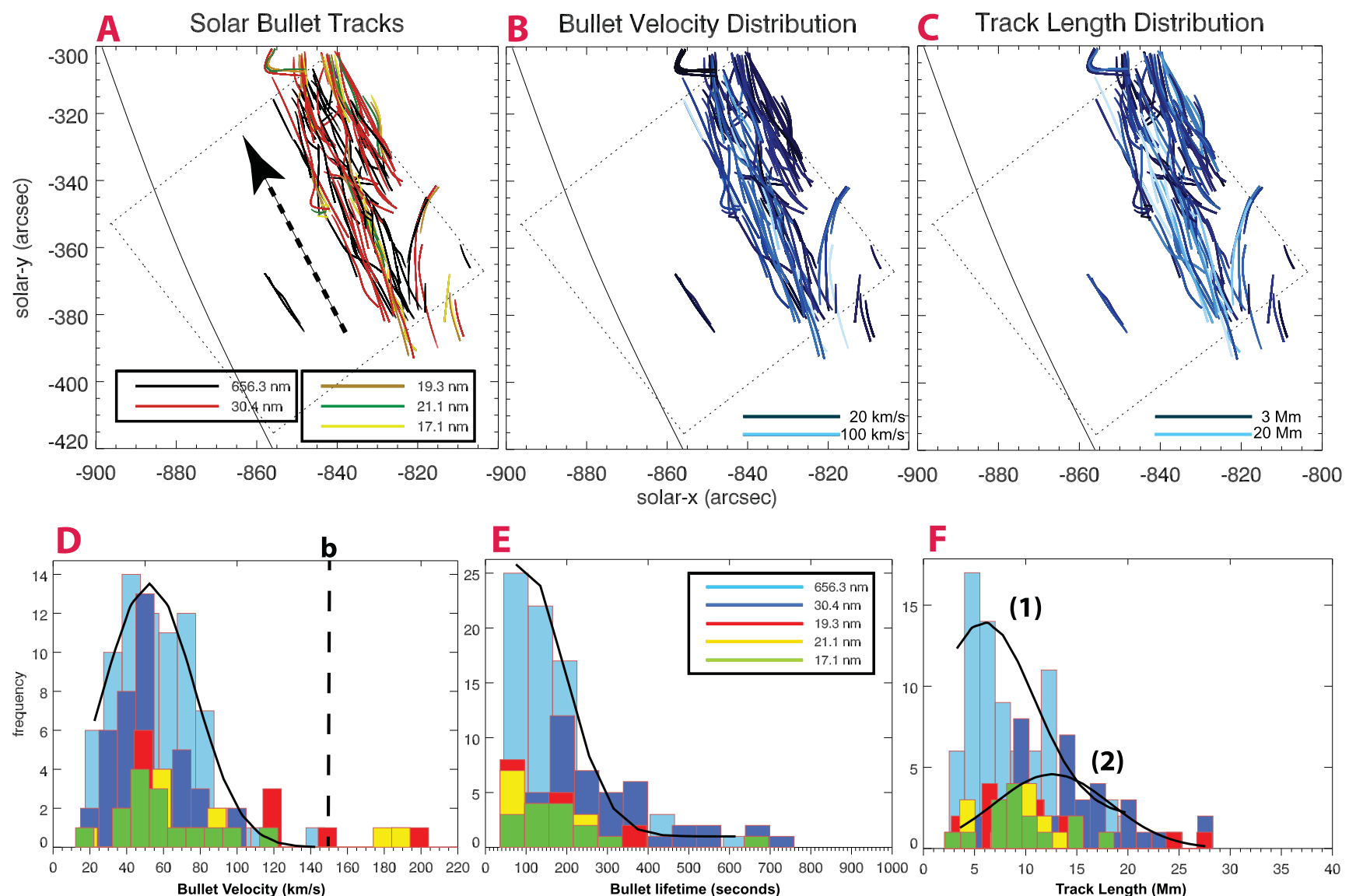

E

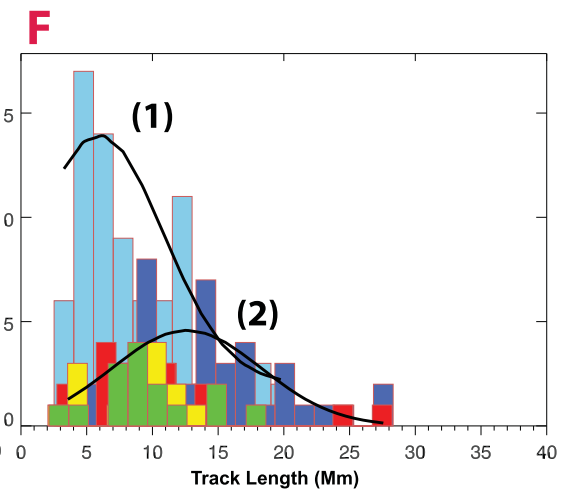

Figure 6. Statistics on all detected bullet tracks identified within the FOV (panel A-color-scale denotes spectral channel) are presented in panels (D-apparent velocity $\left(\mathrm{km} \mathrm{s}^{-1}\right)$, E-lifetime (s), and F) - track length (Mm). Panel B describes the spatial distribution of bullet tracks in a blue color scale, whereby light blue denotes relatively faster bullets and darker bullets denote relatively slower bullets. Likewise, panel C describes the spatial distribution of bullet lengths with light blue depicting the longest lived bullets and progressively darker tracks representing shorter bullet tracks. The faint solid line (panels A-C marks the solar limb in AIA $170.0 \mathrm{~nm}$ and the outline of the CRISP FOV. Panels (D-F) show histograms of the properties of all of the bullets of panel A. A different color scale is used to distinguish the different spectral channels, as shown in the legend of panel E. The fitted Gaussian profile in panel F marked as (2) is fitted to the $30.4 \mathrm{~nm}$ distribution. The vertical dashed line (labelled $b$ ) in panel D, represents the upper limit of bullet velocities within the relatively cool bullets of $\mathrm{H} \alpha$ and $30.4 \mathrm{~nm}$. See the animation associated with this figure for the formation of bullet tracks in $\mathrm{H} \alpha$ and $30.4 \mathrm{~nm}$, collectively, as presented in panel A.

(An animation of this figure is available.)

coincident $\mathrm{H} \alpha$ and $30.4 \mathrm{~nm}$ bullets of thread \#1 extend further across the FOV and then appear to split into another pair of coincident $\mathrm{H} \alpha$ and $30.4 \mathrm{~nm}$ bullets. Extending from this new pair of bullets, an independent $\mathrm{H} \alpha$ bullet is triggered that propagates in the direction of thread \#2, at $\sim 70^{\circ}$ to thread \#1. At 11:04:55 UT $\left(t_{0}+270 \mathrm{~s}\right)$ the new pair of bullets in thread \#1 appears to trigger a new $\mathrm{H} \alpha$ bullet which then continues to extend further along thread \#1 (between panels 3 and 4). The total length of thread \#1 is estimated to be $15.0 \mathrm{Mm}$ from source A. After $90 \mathrm{~s}$, the newly formed $30.4 \mathrm{~nm}$ bullet in thread \#2 appears coincident with a chain of two short-lived $\mathrm{H} \alpha$ bullets. In the same time interval, the $30.4 \mathrm{~nm}$ bullet formed in thread \#2, extends a great distance along thread \#2. The estimated total length of thread \#2 is $16.2 \mathrm{Mm}$.

Only $63 \mathrm{~s}$ before the end of cascade A (11:09:07 UT) do we detect the beginning of cascade B (Figure 7, bottom rows in panels 1-4). Notably, the origin of this cascade (source B) is situated ahead of source A within cascade A and between threads \#1 and \#2. The total duration of cascade B is $271 \mathrm{~s}$, which is approximately half the lifetime of cascade A. Cascade $\mathrm{B}$ is composed of bullets in all wavelengths discussed here and there is only one dominate thread (\#3). Thread \#3 is in fact situated between threads \#1 and \#2 of cascade A, so the formation of cascade B is not entirely disconnected from cascade A. Just as with thread \#1, thread \#3 also appears to originate as a coincident pair of $\mathrm{H} \alpha$ and $30.4 \mathrm{~nm}$ bullets and in its progression it also triggers bullet tracks in directions nearorthogonal to the predominant direction of the cluster. Cascade B contains a bullet network that is more compact and contains a higher number density of short-lived bullets, compared to cascade A. Thread \#3 has a total length of $9.8 \mathrm{Mm}$ and contains a total of 14 individual bullets. Just as with thread \#2, the initial pair of bullets within thread \#3 evolves up the temperature spectrum within $91 \mathrm{~s}$, to apparently trigger even hotter 17.1 and $21.1 \mathrm{~nm}$ bullets. Then, $90 \mathrm{~s}$ after this, the hot bullet cluster appears to trigger a burst of very-short-lived cool $\mathrm{H} \alpha$ bullets, as seen in Figure 7 (bottom rows of panels 3 and 4). Just as with cascade A, the evolution of bullet tracks in cascade B appears to fan out across the FOV, away from the source region. Cascade B ends at 11:13:38 UT, less than two minutes prior to the start of the largest cascade in the cluster, i.e., cascade C.

\subsection{Cascade $C$}

Figure 8 presents the largest detected cascade within the cluster. Cascade $\mathrm{C}$ is also composed of two threads (\#1 and 
CASCADE A

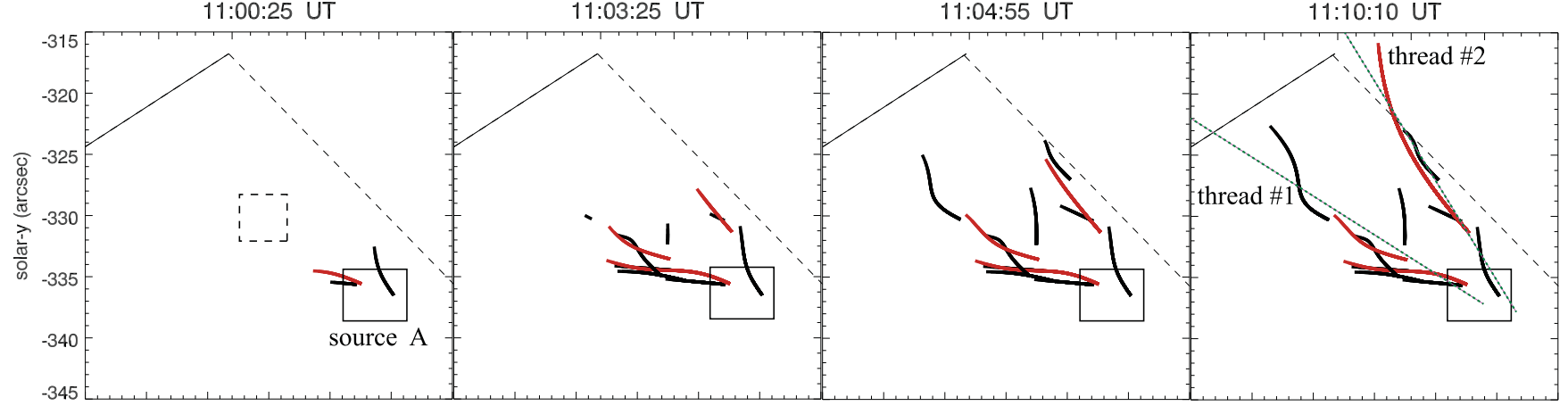

\section{CASCADE B}

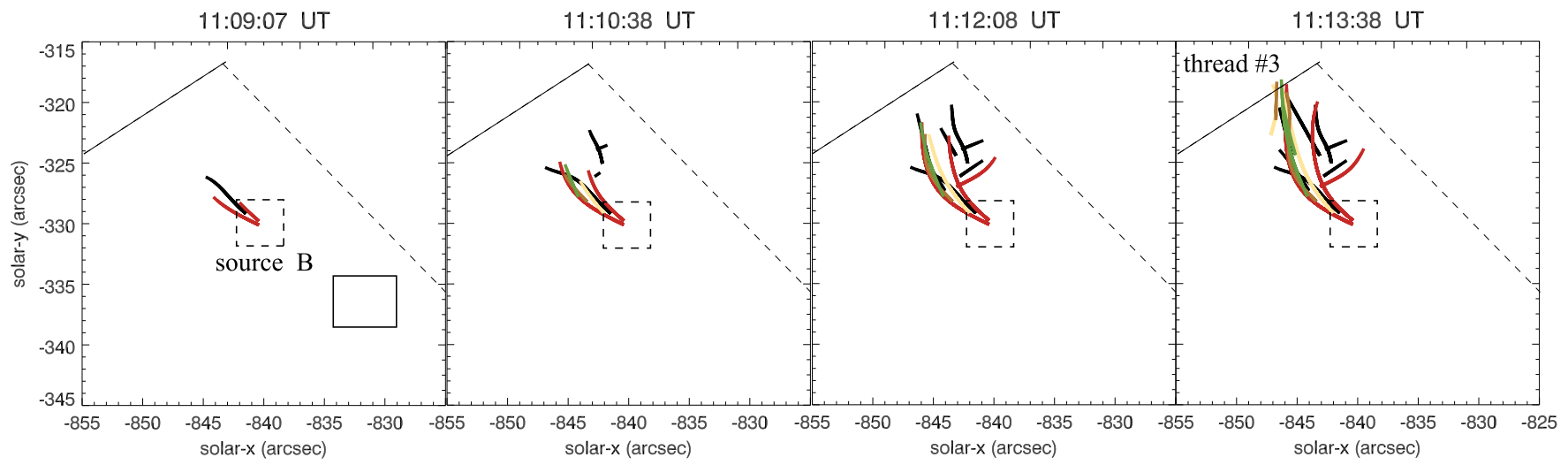

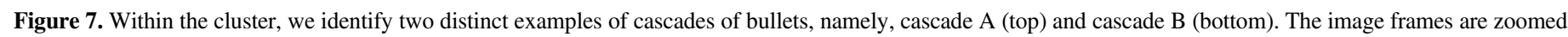

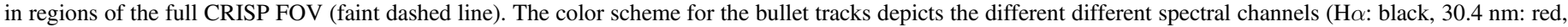

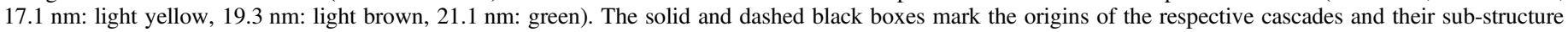
threads \#1, \#2 (cascade A), and \#3 (cascade B). See the animations associated with this figure for movies of cascades-A and B.

(Animations $\mathrm{a}$ and $\mathrm{b}$ of this figure are available.)

\#2), the second of which contains the highest number density of bullets (28) in all wavelengths. Thread \#1 (top row) appears later in time than thread \#2 (bottom row) and contains 16 bullets. The larger spatial extent of bullets in thread \#1 allows us to investigate the dynamic interactions between bullets in the cascade progresses. ${ }^{3}$ Thread \#2 has a range of $67.1 \mathrm{Mm}$, which is more than double that of thread \#1 with a range of $31.2 \mathrm{Mm}$. Within the range of the cascade, there are several interesting source regions where the apparent coronal line bullets appear in the FOV, labelled A1-A2 followed by B1-B2 then $\mathrm{C} 1-\mathrm{C} 2$ (corresponding to their order of appearance). The order of appearance for all bullets is presented in Figure 8 panel 2 for both threads (rows), and again there is a clear evolution from bottom right (light blue) to top left (progressively darker blue). Despite thread \#2 having $>50 \%$ range of motion compared with thread $\# 1$, its duration is only $\sim 9 \%$ greater. There is an indication of a higher average group velocity in the bullets of thread \#2 compared to thread \#1. Thread \#2 appears before thread $\# 1$ and has a higher than average group velocity of $66 \mathrm{~km} \mathrm{~s}^{-1}$.

Source regions here are identified as regions of interaction/ formation of the hottest bullets. Given that they occur sequentially within the progression of the cluster (from the order of appearance of the bullets for threads \#1 and \#2) this implies that they may not be independent events. The sources

\footnotetext{
3 See the animations associated with Figures 6-8 for the evolving bullet tracks in all cascades presented.
}

coincide with the formation of cooler bullets, which extend and expand the cluster across the FOV. For confirmation of the correspondence between hot and cool bullets, we present the $X-T$ diagrams for source B2 trigger event in Figure 4 (bottom row). Through aligning the flows within the green-dashed line, in each $X-T$ diagram, it can be shown that the initially hot motion of the bullet (in AIA 17.1, 19.3, $21.1 \mathrm{~nm}$ ) are followed later in time by an increased intensity and formation of the $\mathrm{H} \alpha$ counterpart, within the $225 \mathrm{~s}$ total lifetime of the bullets' trigger event. This direction of evolution of sources is indicated with green arrows in panel 2 of thread \#2 (Figure 8). The separation angle between the global progression of the cluster of all bullets (black arrow) and the signal inferred from the evolution of the trigger events is $35^{\circ}$.

\subsection{Cascade Trigger Events}

A set of the highest velocity bullets (in the range of $85-200 \mathrm{~km} \mathrm{~s}^{-1}$ ) exist in Figure 6 outside (or at least on the tailend) of the Gaussian distribution fitted for $\mathrm{H} \alpha$ bullets. We can confirm that these high velocity bullets correspond to the location of the trigger events of cascade $\mathrm{C}$ threads \#1 and \#2. The trigger events contain only the apparent coronal line bullets and precede cooler bullet formation (in $\mathrm{H} \alpha$ and $30.4 \mathrm{~nm}$ ) in thread \#2 (as mentioned previously). The bullet tracks for trigger events are distributed across the FOV, as shown within the sub-plots of Figure 9. In the left sub-plot, there is a direct connection between hot bullets (in 30.4, 17.1 and $19.3 \mathrm{~nm}$ ) 

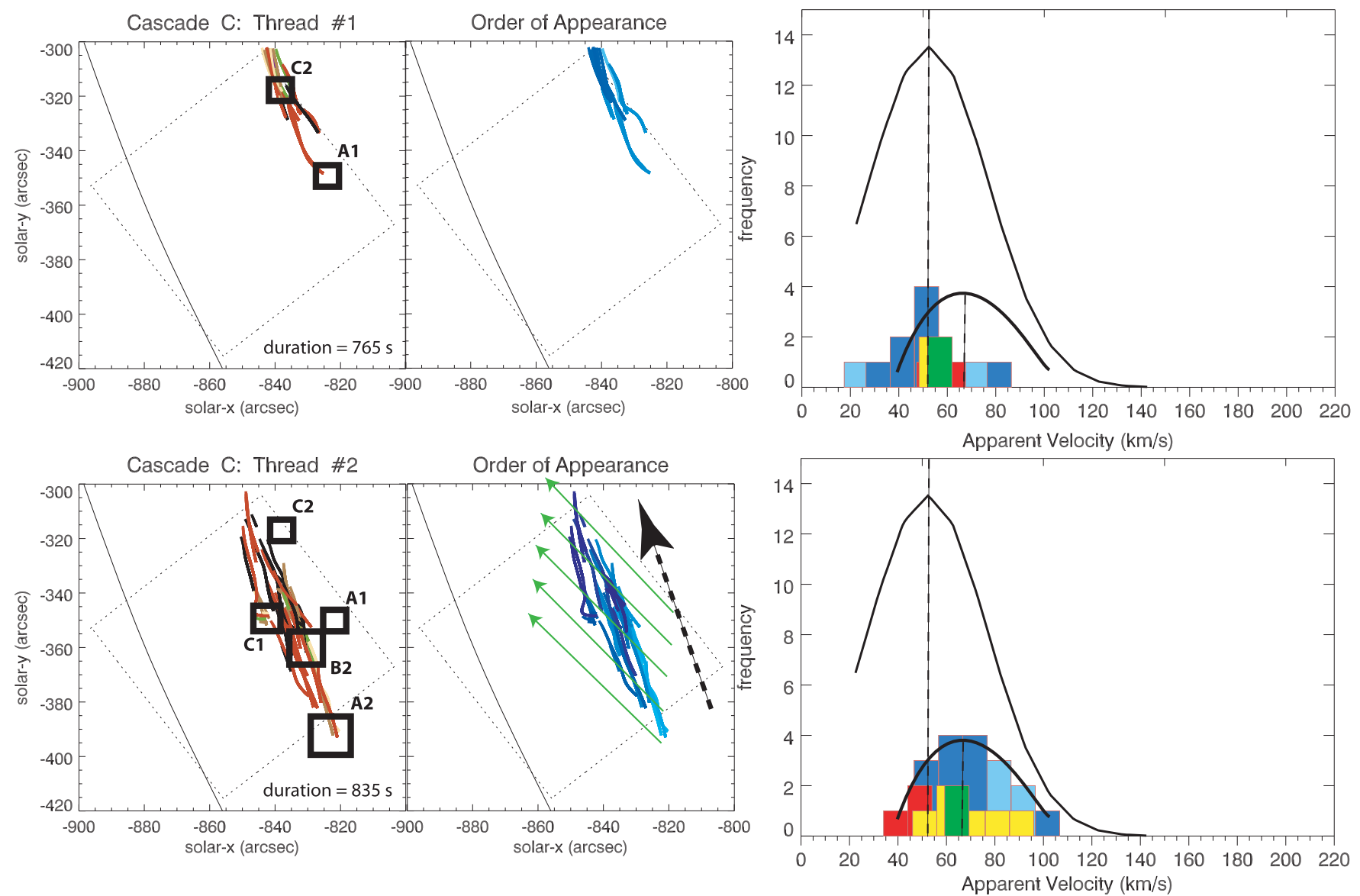

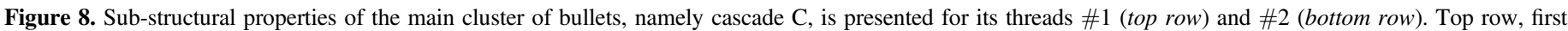

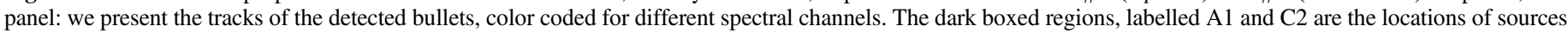

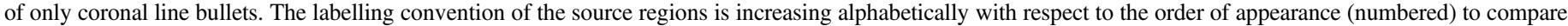

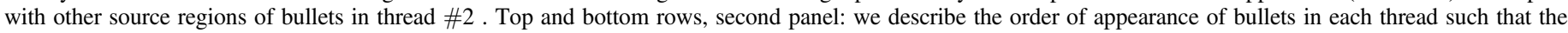

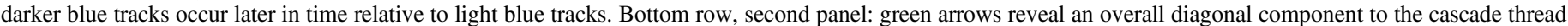

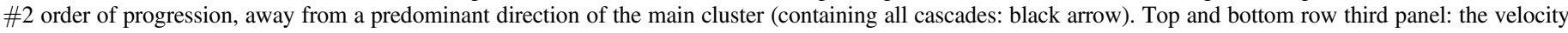
distribution of bullets in each thread of the cascade in comparison with the Gaussian distribution for all bullets. See this figure's animation of cascade-C.

(An animation of this figure is available.)

from the initial source A2 of thread \#1 in cascade C and source B2 bullets. In the right sub-plot, the order of appearance also depicts the sequential evolution of the trigger bullets, i.e., numbered from 1-10 (light blue to dark blue) in thread \#1. The bullet tracks have the same direction so there is no direct association between hot bullets of source B2 and source $\mathrm{C} 1$. The hot bullets corresponding to source $\mathrm{C} 1$ occur in sequence after A2 then B2. Cooler bullet tracks are shown to evolve out of all of the source regions, which have lower velocities on average compared to the hot high velocity trigger bullets. These connecting bullets linking sources $\mathrm{C} 1$ with $\mathrm{B} 1$, as well as $\mathrm{C} 2$ with B2 and A1 (of thread \#1), can be more easily seen in Figure 8 (panel one, bottom row), via the tracks from the cooler channels of $30.4 \mathrm{~nm}$ and $\mathrm{H} \alpha$. This gradual progression of cooler bullet tracks appearing out of the hottest source regions, as also shown in Figure 10. The green dashed line in Figure 10 marks the interaction region between the relatively hot red $(30.4 \mathrm{~nm})$ and relatively cooler black $(\mathrm{H} \alpha)$ tracks. These tracks correspond to the longest lifetime bullets detected here, which are greater than $400 \mathrm{~s}$.

Aside from evidence of coincident $\mathrm{H} \alpha$ and $30.4 \mathrm{~nm}$ bullets in cascade $\mathrm{A}$, there is clearly evidence of greater temporal thermal evolution in the detected progressive cooling of consecutive bullets in cascade B and C. Another characteristic of bullet evolution is with respect to their direction of propagation discussed next.

\subsection{Bi-directional Bullets}

One particularly interesting finding in this analysis may be the discovery of bi-directional bullets in $\mathrm{H} \alpha$ and $30.4 \mathrm{~nm}$ channels. Six examples where found and four are presented in Figure 11. In all but one case, the $30.4 \mathrm{~nm}$ bullet travels in the upward direction following the cascade, with an associated $\mathrm{H} \alpha$ bullet motion in almost exactly the opposite direction, and both are initiated from a common origin. The other important difference in each example is the delay interval between the bidirectional bullet formation in $\mathrm{H} \alpha$ and $30.4 \mathrm{~nm}$. The longest delay corresponds to $\sim 108 \mathrm{~s}$ with respect to the start time of the bullet in $\mathrm{H} \alpha$ versus $30.4 \mathrm{~nm}$. However, there is one instance where the bi-directional bullets occur within the same time frame so the delay is less than $9 \mathrm{~s}$ (the CRISP cadence) making this very much co-temporal. The source region corresponding to each example, reveals that the spatial offset between the start of oppositely directed bullets is always within the range of $2^{\prime \prime}-$ 7 " on average. The origins of the bi-directional motion appear 


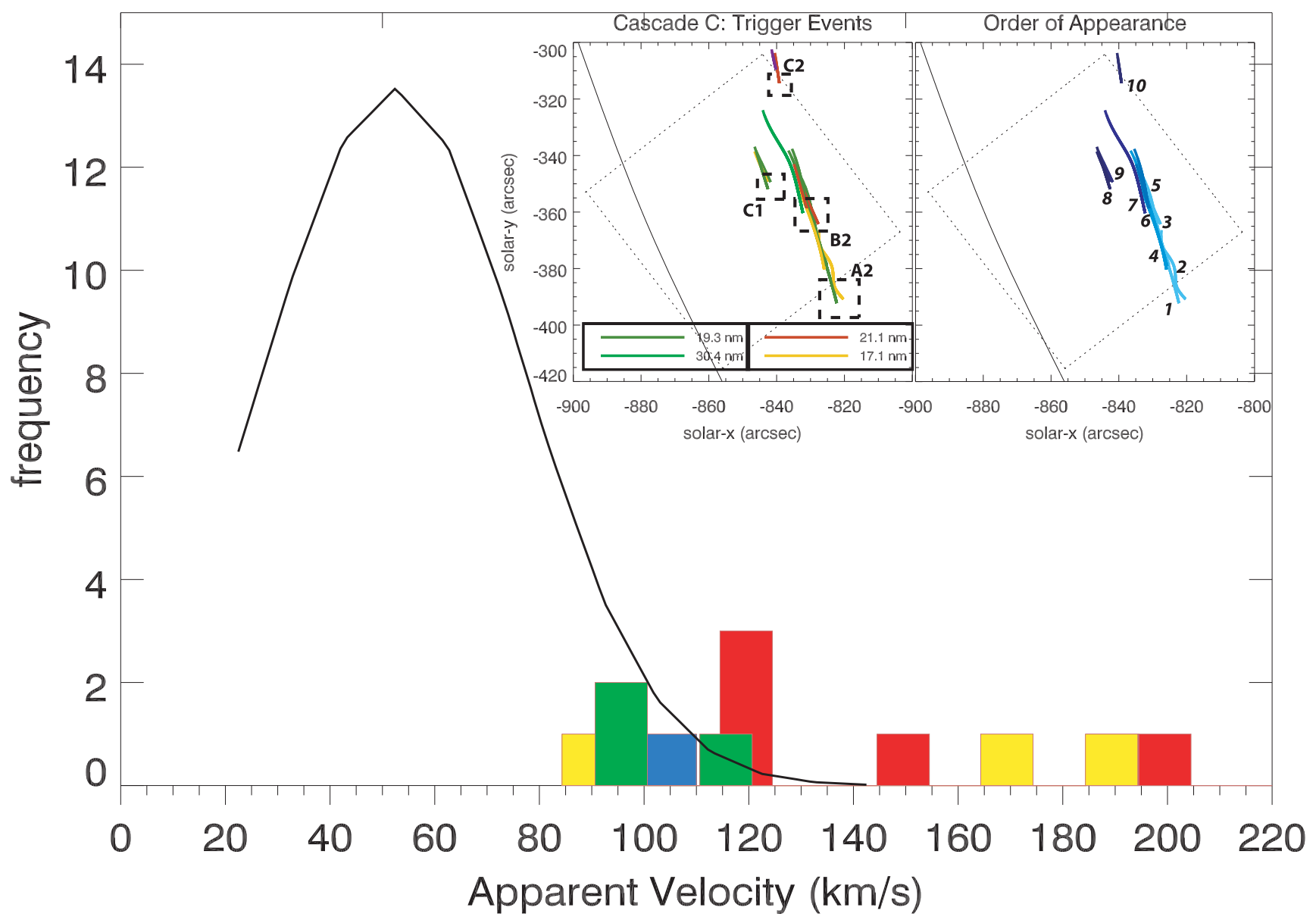

Figure 9. Highest velocity and hottest bullets from cascade $\mathrm{C}$ trigger events $(A 2-C 2)$ are: (a) reproduced as color-coded tracks in the sub-plots (Left: spectral distribution, Right: numbered order of appearance) and (b) displayed on the color-coded bullet velocity histogram, which is over plotted with the H $\alpha$ bullet Gaussian-fit (black curve). See the animation associated with Figure 11, which shows one example of a strong association between a bullet formation and an explosive trigger event.

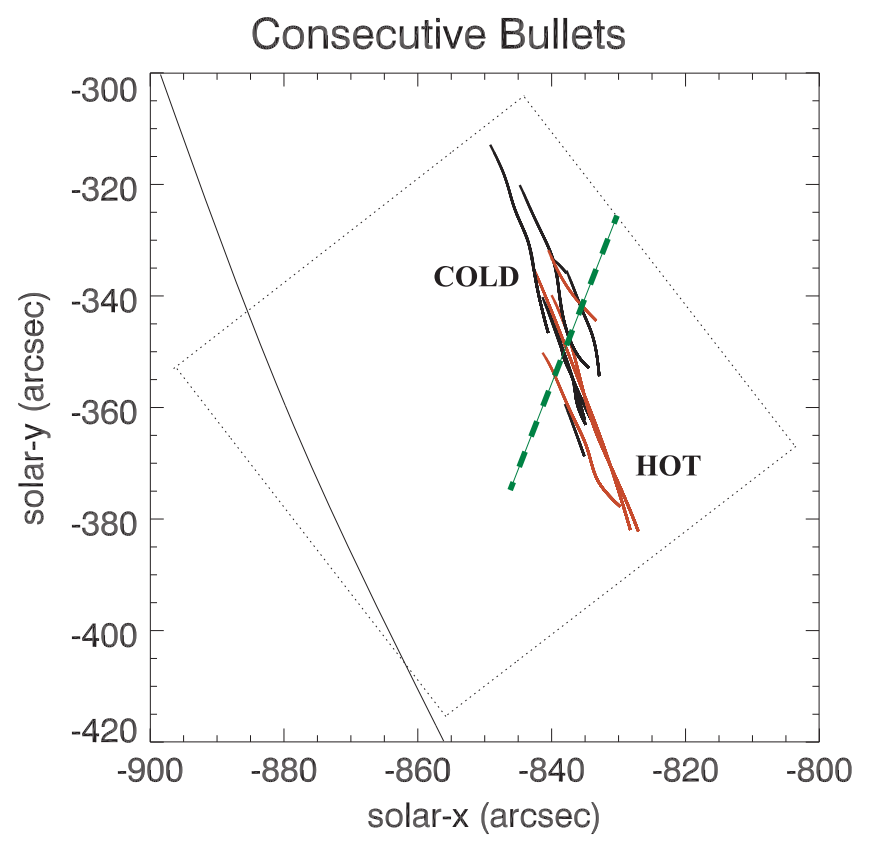

Figure 10. Lower velocity, cooler bullet tracks of cascade $\mathrm{C}$ are presented. $\mathrm{Ho}$ (black) and $30.4 \mathrm{~nm}$ (red) bullet tracks of thread \#2 are compared. The bullets appear to propagate consecutively from the bottom right to the top left almost entirely across the CRISP FOV (outlined). The green dashed line indicates the interaction region marking the transition from relatively warm to cool bullets. within the boxed regions, which lie close to the sources of trigger events driving the cluster cascade-C. Although at the time of their occurrence there is no clear explosive activity within that might suggest a hot "trigger" origin to the outward propagating bullets. This behavior may provide valuable information on the nature of the formation of bullets.

\section{DISCUSSION}

This paper represents a more in-depth, statistical analysis of a cluster of small-scale, bright blobs (now referred to as bullets), as initially described in Paper I. A new analysis of the cluster here has provided new insights into the dynamics of bullets, as well as their thermal structure. This was achieved through the incorporation of new information from EUV channels via SDO/AIA observations sampling at least TR temperature plasma in bullets. Co-spatial and co-temporal activity from AIA, together with $\mathrm{H} \alpha$ bullets in CRISP provide valuable new spectral information.

\subsection{New Observable Characteristics of Bullets}

Bullets appear as small-scale, bright, laterally propagating projectiles that follow magnetic field topology, tend to appear in cascade, and are self-contained thermally evolving structures. The foremost result in this research is the one-to-one correspondences of bullets observed in a broad range of 


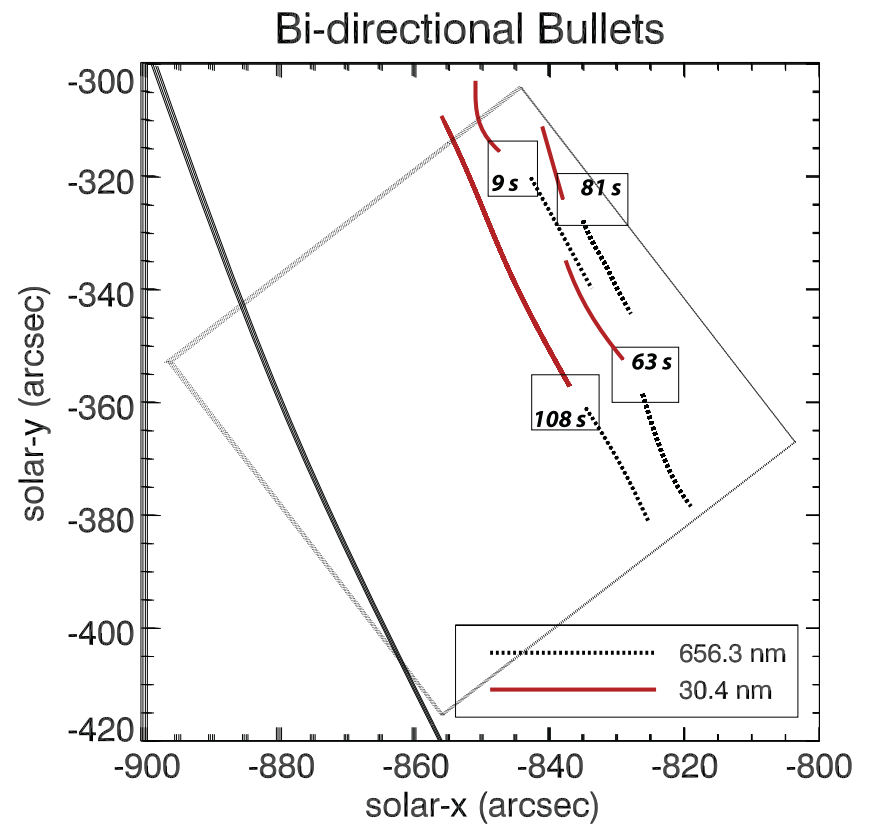

Figure 11. Bi-directional motion of bullets are evident in six examples, four of which are presented here, for bullets observed in $\mathrm{H} \alpha$ and $30.4 \mathrm{~nm}$. The solid line tracks correspond to bullet motion only in the upward direction in the FOV, i.e., in agreement with the general cascade direction. The dashed-line tracks correspond to bullet motion only in the downward direction in the FOV. The boxed regions indicated the sources of each bullet pair in these examples. The delay intervals printed in each panel correspond to the offset in the start time of the bullets in their respective channels.

(An animation of this figure is available.)

spectral channels. Bullets can evolve in a number of ways throughout their lifetime. Some of the defining characteristics of bullets are as follows.

Size and Brightness: It is important to consider the reduced spatial resolution of the AIA instrument when observing $30.4 \mathrm{~nm}$ in comparison with the more resolved $\mathrm{H} \alpha$ bullet components from CRISP. We tracked a total of 171 bullets within a dynamic, propagating cluster. More than half of these where observed in $\mathrm{H} \alpha$. The features appear close to the diffraction limit within the CRISP observations. We measured typical bullet widths, using boundaries at the 10\%-20\% intensity level (relative to the background), to be in the range of $0 . " 3-0.4$, corresponding to $215-290 \mathrm{~km}$, which is in agreement with Paper I. Alexander et al. (2013) presented observations of numerous small-scale anti-parallel flows within coronal loop threads in the $19.3 \mathrm{~nm}$ coronal channel at a resolution limit close to that of Hi-C (Cirtain et al. 2013). It was concluded that such fine scale million degree structures would have gone undetected in AIA. Therefore, it may be that the $30.4 \mathrm{~nm}$ bullets observed in AIA here could also exist at scales more comparable with the $\mathrm{H} \alpha$ bullets. Zacharias et al. (2011) simulated the formation of a small-scale (1-2 Mm) hydrodynamic blob-like feature numerically, which was seemingly pumped from a loop foot-point in the solar transition into the corona along a trajectory defined by the coronal magnetic field. The observed bullets described here differ, such that they are almost one order of magnitude smaller, they fragment into a cluster, are faster, and have a more complicated thermal structure. We discovered that some bright bullets in $\mathrm{H} \alpha$ are cospatial and co-temporal (coincident) with similar features in emission in $30.4 \mathrm{~nm}$; however, the $30.4 \mathrm{~nm}$ bullet, on average, has a larger diameter of $1-1$ ". 5 or $720-1080 \mathrm{~km}$. The size ratio of coincident bullets can vary quite a lot. Bullet 3 of Figure 3 panel $\mathrm{C}$ has a $30.4 \mathrm{~nm}$ counterpart that is $\sim 15$ times larger in cross-sectional area. One assumes that the $\mathrm{H}$-alpha bullet represents the cool, compact core being surrounded by a slightly larger $30.4 \mathrm{~nm}$ emitting TR. The true size of the $30.4 \mathrm{~nm}$ bullet cannot be determined from the current data, given the instrumental limitations.

The relative brightness ratio (with respect to the spectral background level) was a factor of $\sim 2$ greater in the $30.4 \mathrm{~nm}$ bullet than in the coincident $\mathrm{H} \alpha$ bullet. The relative intensity of $\mathrm{H} \alpha$ bullets appears within a 10\%-20\% intensity range while the coincident $30.4 \mathrm{~nm}$ bullets have a 50\%-60\% intensity range throughout the cluster (as depicted in Figure 2 sub-plot [2]). The intensity ratios determined here are measured with respect to the background level of the respective passbands of $30.4 \mathrm{~nm}$ and $\mathrm{H} \alpha$. However, the brightness ratios in these lines are widely different in terms of radiated energy per unit area and time. The background continuum around $\mathrm{H} \alpha$ is about 300 times brighter than the observed background of $30.4 \mathrm{~nm}$ (Lean 1991; Woods 2002). Given that the $\mathrm{H} \alpha$ line center is close to $20 \%$ of the nearby continuum level suggests that the $\mathrm{H} \alpha$ reference background could be 60 times that of the $30.4 \mathrm{~nm}$ background. Taking into account the factor of two difference in the relative intensity ratios of the bullets deduced here, on an absolute scale, it should correspond to $\mathrm{H} \alpha$ bullets being around 30 times brighter that the $30.4 \mathrm{~nm}$. As an approximation, the $30.4 \mathrm{~nm}$ bullets may indeed be at least a factor of 10 brighter relative to the background than actually observed at the resolution of AIA. Therefore, taking this factor of 10 into consideration for the bullet case, the best estimate is that the typical absolute brightness of the $\mathrm{H} \alpha$ bullets is 3-10 times brighter than the corresponding $30.4 \mathrm{~nm}$ bullet. Hall \& Hinteregger (1970) and Ahmad \& Withbroe (1977) indicate a relative difference of several orders of magnitude in considering $\mathrm{H} \alpha$ compared with $30.4 \mathrm{~nm}$. This estimate seems reasonable since the $30.4 \mathrm{~nm}$ component is possibly emitted from a thinner, surrounding transition layer. The picture being presented here is of a bullet with a highly rounded 3D structure, a relatively dense and cooler core (in $\mathrm{H} \alpha$ forming at $\sim 7000-10,000 \mathrm{~K}$ ), which has an outer envelope of transition region of less dense and hotter plasma at $\sim 80,000 \mathrm{~K}$.

Brightness evolution: The predicted existence of a temperature evolution of the $\mathrm{H} \alpha$ bullets from Paper I, is confirmed in this study. The majority of bullets tracked appear as bright $\mathrm{H} \alpha$ features with one-quarter of all tracks observed in $30.4 \mathrm{~nm}$, and about one-eighth were equally distributed across the hotter channels. A very interesting finding in comparing coincident $\mathrm{H} \alpha$ and $30.4 \mathrm{~nm}$ bullets is the inverse relationship in the brightness of these two lines as shown in the sub-plot [2] of Figure 5, with a fitted gradient of -2.18 despite the linear correlation between corresponding/coincident bullets with respect to velocity. Assuming that the $\mathrm{H} \alpha$ line emission is controlled by recombination in a partially ionized hydrogen gas and thereby scaled as $N_{e}^{2}$ (cf Engvold 1976), the observed variations in $\mathrm{H} \alpha$ emission should imply fairly modest changes in density of about 3\%-5\%. A momentary increased emission in $\mathrm{H} \alpha$ will result from an electron density increase, which subsequently leads to increased recombination and enhanced Balmer line emissions. The effect on the $30.4 \mathrm{~nm}$ intensity resulting from a density increase will be different, as shown in non-LTE prominence model calculations (Labrosse \& 
Gouttebroze 2001; Labrosse et al. 2010). A density increase will lead to a decrease in singly ionized $\mathrm{He}$, which very likely also may result in a decrease in $30.4 \mathrm{~nm}$ emission. The sub-plot [2] of Figure 5 is in agreement with this interpretation since increased brightening in $\mathrm{H} \alpha$ (implying electron density increase) corresponds with reduced intensity in $30.4 \mathrm{~nm}$ matched bullets. Since the $30.4 \mathrm{~nm}$ line is also influenced by incident radiation from the surrounding atmosphere, further discussion of the observed inverse relation should be based on more detailed radiative transfer modeling. Bullet formation could be interpreted as a response of an AR corona being in a near-continuous state of thermal non-equilibrium, leading to recycling of thermal energy transferred in the form of bullets. Evidence for the effects of thermal non-equilibrium in the form of small-scale coronal loop sub-structures is clearly demonstrated in recent advanced 3D radiation MHD simulations (Olluri et al. 2013; Froment et al. 2014). The detectability of the bullets in the hotter EUV channels, such as 19.3 and $21.1 \mathrm{~nm}$, is more challenging given the reduced signal-to-noise and apparently more diffuse appearance of the bullets, within the respective $X-T$ diagrams. As a consequence of the intensity ratio thresholding, as a criteria for bullet selection, we may be under-sampling the bullet number densities detected in these channels. The response of various emission lines in the EUV to the bullet triggering mechanism, will inevitably depend on the radiative origin and nature of these different lines, which is further complicated by the broadness of the passbands and thereby sensitive to line blending. We have already indicated that $\mathrm{H} \alpha$ of neutral hydrogen responds very differently compared with He II $30.4 \mathrm{~nm}$ of ionized helium. It is a very important issue for future follow-up studies to understand the significance of the relative brightness variations in all other EUV channels presented here, with regard to the multithermal nature of bullets.

Velocity properties: Bullets propagate with relatively constant velocities in time. From Figure 6, we find that the bullet velocity distribution in the cluster peaks at $52 \mathrm{~km} \mathrm{~s}^{-1}$ with an FWHM of $+/-33 \mathrm{~km} \mathrm{~s}^{-1}$. This distribution is again in agreement with the velocity properties of Paper I; however, now we can demonstrate an equally close agreement with the hotter bullets in the cluster, as observed with AIA. Within the $\mathrm{H} \alpha$ bullet velocity distribution of Figure 5, we find a striking linear correlation with coincident $30.4 \mathrm{~nm}$ bullet velocities, as presented in sub-plot [1], with a fitted line with 0.87 gradient indicating an almost 1:1 linear correlation between the bullets. The tail-end of the velocity distribution in $\mathrm{H} \alpha$ extends out to a maximum of $145 \mathrm{~km} \mathrm{~s}^{-1}$, which corresponds to the fastest detected $\mathrm{H} \alpha$ bullet. However, an interesting feature from the velocity distribution was the presence of very-high-velocity bullets, which exist outside of the Gaussian fit to the more abundant cooler bullets. When considering only the AIA channels, the fastest bullet detected was in the apparent coronal $19.3 \mathrm{~nm}$ channel, with an apparent velocity of $200 \mathrm{~km} \mathrm{~s}^{-1}$. Higher velocity bullets appear early on in the evolution of the cluster with progressively cooler bullets following. This trend was in agreement with the order of appearance of cascade $\mathrm{C}$ (Figure 8). Within the cluster, we identified a number of sources of concentrated bullet tracks from the apparent coronal channels, named as trigger events. These trigger bullets propagate in the range of $85-200 \mathrm{~km} \mathrm{~s}^{-1}$. Within the main cluster 5 trigger source regions could be identified and they spread sequentially across the main cluster. Note that there is no observational evidence for Doppler shifts of the bullets in $\mathrm{H} \alpha$, where we have highly sampled line scans.

Cascading trajectories: As the cascade evolves, the bullets appear to fan outward into multiple threads in what could be described as an avalanche-effect. $63 \mathrm{~s}$ before the end of cascade A (11:09:07 UT), we detect the beginning of cascade B. Additionally, thread \#3 is situated between threads \#1 and \#2 of cascade A, so the formation of cascade B is most likely related to cascade A. The vast majority of the detected bullets propagating along the same direction within the FOV (represented by the large black arrow in Figure 6) and throughout the evolution of the cascades. Within individual bullets there are slight deviations away from the straight-line direction, as is evident from the threads of cascade A in Figure 7. Bullets do not necessarily propagate in one given direction over their lifetime. $\mathrm{H} \alpha$ Bullet \#3 of Figure 3 (panel C) displayed a significant deviation to the left (in the plane of sky) by $\sim 70^{\circ}-80^{\circ}$ and then to the right by $\sim 70^{\circ}-80^{\circ}$ (within the white dashed box) to bring it back on course with the cluster flow direction. Nano-flare storms are considered to be one such impulsive heating mechanism that can spontaneously occur in coronal loop systems, driven by multiple small-scale magnetic reconnections and with resulting small-scale projectiles that are thought to exhibit cascade-like behavior (Warren et al. 2002, 2003; Winebarger \& Warren 2005; Klimchuk 2006; Testa et al. 2014). In the case of bullets, we see parallels with nano-flare storms since the cascading and bi-directional bullets are sourced to a common origin in the cluster and therefore may have a common triggering mechanism. Individual nanoflares in coronal loops have been shown to exhibit bursty behavior in $S D O$ channels lasting $500 \mathrm{~s}$ and with lengths of $150 \mathrm{Mm}$ (Viall \& Klimchuk 2012). When we combine the information from the track lengths of cascade $\mathrm{C}$, we estimate, similarly, a total propagation distance of the cluster to be 98.3 Mm over a lifetime of approx. 900-1000 s. Viall \& Klimchuk (2012). Likewise, Archontis \& Hansteen (2014) used radiation MHD simulations of interacting magnetic bipoles in a coronal environment, which resulted in clusters of small-scale flares $1-2 \mathrm{Mm}$ in scale. This activity formed naturally via patchy reconnection, leading to small-scale plasmoid ejections with energetics characteristic of nano-flare behavior. These flares were short-lived ( $30 \mathrm{~s}$ to 3 minutes) and on a similar spatial and temporal scale, as hot components of bullets observed here. However, those simulated plasmoids exhibit similar characteristics from previous studies of larger flare-driven plasmoid eruptions (Tsuneta 1997; Archontis et al. 2006), such as, having associated jets and cusp-like structures and we do not detect such flaring behavior associated with bullets here. Furthermore, the cluster of bullets tend to laterally propagate and cascade across the FOV, rather than erupt upward or downward. Since there is no evidence for Doppler motions in bullets, we do detect evidence of internally heated mass flows in bullets and so they cannot be similar to nano-flare behavior.

Lifetime: The peak in the lifetime distribution for the cluster (Figure 6) revealed that most bullets exist for $75 \mathrm{~s}$ with an HWHM extending the typical lifetimes of bullets to $135 \mathrm{~s}$. The hottest (19.3 and $21.1 \mathrm{~nm})$ detected bullet lifetimes are in the range of 35-95 s (with peaks at $65 \mathrm{~s}$ in $21.1 \mathrm{~nm}$ and $70 \mathrm{~s}$ in $19.3 \mathrm{~nm}$ ), whereas, the relatively cooler 17.1 and $30.4 \mathrm{~nm}$ bullets have a peak lifetime in the 155-215 s interval. Given that the left-side tail of the lifetime Gaussian distribution is not 
present in Figure 6 (panel E), implies that there may be shorterlived bullets that have gone undetected. With a detection criteria of at least six frames used to construct bullet tracks (i.e., making them identifiable within $X-T$ diagrams), the shortestlived detectable bullets will therefore have a lifetime of $\sim 54 \mathrm{~s}$, so our observations are not very sensitive to short-lived bullets of less than 1 minute. Faster bullets with shorter lifetimes (than the minimum lifetime detected of $69 \mathrm{~s}$ ) should be detected within our detection criteria of six frames. If they exist, they are presumed to have been too faint to confidently detect for this study. One notable feature concerning $30.4 \mathrm{~nm}$ and $\mathrm{H} \alpha$ bullets is the difference in the observed lifetimes and lengths with $30.4 \mathrm{~nm}$ being on average longer lived by $\sim 100 \mathrm{~s}$ resulting in their longer lengths on average (as revealed in distributions (1) and (2) of panels E and F of Figure 6). This could be a result of differing rates of compression of the plasma, assuming an MHD fast mode pulse explanation, for the same reason as discussed in the brightness evolution section. As the MHD pulse amplitude may gradually be dampened and weaken and thereby decrease the induced plasma density, it will lead to some reduction in the $\mathrm{H} \alpha$ bullet emission. The consequences for the corresponding $30.4 \mathrm{~nm}$ bullet may be different for reasons discussed under the subsection of brightness evolution, which ultimately may give rise to slightly different bullet lifetimes in these two spectral lines.

Multithermal structure: Co-moving, multithermal associations of bullets most likely imply that bullets are wrapped in a temperature transition layer. Such properties have been observed previously in the outer solar atmosphere, e.g., in the envelope that appears around large-scale filaments, commonly referred to as the prominence-corona-transition-region (PCTR: Chiuderi \& Chiuderi Drago 1991; Heinzel et al. 2001; Heinzel \& Anzer 2001; Labrosse et al. 2010). In the PCTR models, the temperature of plasma in prominences exhibits a similar rise from about $7000 \mathrm{~K}$ up to 1 million $\mathrm{K}$. Given the relative proximity of the formation of the $\mathrm{H} \alpha$ line core (Leenaarts et al. 2012) and the $30.4 \mathrm{~nm}$ line, with respect to height in the solar atmosphere, it is expected that there should be a lot of correspondence between the behavior of detectable features in both spectral channels. The AIA instrument has a broad temperature coverage in the $30.4 \mathrm{~nm}$ channel compared with the narrower thermal range of the $\mathrm{H} \alpha$ line core with CRISP. Assuming an environment where there is a local pressure and thermal equilibrium, as an initial condition, a change in density will be accompanied by a local change in pressure and emission and so detectability in cooler spectral channels. This imbalance of thermal equilibrium, with respect to temperature and density fluctuations, may be evident in the examples of bullet threads in cascades A and B (see Figure 7), which appear to fluctuate between the $30.4 \mathrm{~nm}$ and $\mathrm{H} \alpha$ passbands. The important point here is that in describing the multithermal structure, the bullet itself may not have necessarily ended at the apparent end-point along its track but rather simply evolved out of detection in that respective passband, as a result of local internal enhancements in pressure/density which explains the observed brightness variations, rather than by heating or cooling processes.

\section{CONCLUSIONS}

This study highlights the fact that the outer atmosphere of the Sun is riddled with small-scale, dynamic and rapidly evolving structures that frequently appear out of thermodynamic equilibrium. The most notable new result in this analysis is the discovery of multiple, connected bullet cascades and bi-directional motions in the activity of a laterally propagating cluster of bullets, previously studied as part of Paper I. The observations of cascades of bullets at high resolution, across a broad temperature spectrum is a very new and exciting insight into the fine-scale structure of the solar corona and transition region. Given that many of the bullets appear close to the diffraction limit in CRISP H $\alpha$ spectral scans and that we have set a fixed intensity contrast criteria for selection in the $X-T$ diagrams, we conclude that the number densities of bullets detected in each of the wavelength channels must be considered as a lower limit. The nature of solar bullets remains open to debate and in our conclusions, we will outline the pros and cons concerning their association with other detections of equally small-scale structures in solar corona AR.

\subsection{Formation of Bullets: Internally Heated Mass Flows or MHD-Type Pulses Affecting Plasma Emission?}

We arrive at two main scenarios depicting their formation. The timescale of multithermal bullet evolution (over hundreds of seconds) is comparable to other similar thermal instabilities, driving small-scale cooling processes with origins near coronal loop-tops, such as coronal rain (see Antolin \& Rouppe van der Voort 2012, and references therein). Such dynamic multithermal behavior in small-scale features near coronal loop-tops of AR, including the short-term presence of chromospheric plasma sub-structures is also attributed to flare-driven coronal rain clumps undergoing catastrophic cooling processes (Scullion et al. 2014). Although, gravity driven acceleration is thought to explain coronal rain flows along magnetic strands, this is not likely in the case of faster moving bullets, which do not appear to fall into the Sun but rather traverse laterally across its surface. Additionally, this picture does not fit because internal heating should evidently cause an observable tail of radiative cooling of the plasma, as detected in coronal rain flows. However, bullets appear as rather distinct, bright slightly elongated structures that do not exhibit tail-like structures.

In Paper I, the MHD wave pulse mechanism was suggested to explain the formation of the bullets. An acoustic (sound pulse) such as the MHD slow-mode could not explain the high speeds of bullets observed. A pure Alfvén wave pulse would not lead to compression of the plasma to account for internal density and temperature variations leading to enhanced emission in the $\mathrm{H} \alpha$. Zaqarashvili et al. (2010) observed an upward propagating intensity blob at the solar limb with similar morphology to bullets described here and proposed that it could be explained as a slow sausage soliton (see Roberts \& Mangeney 1982; Ballai et al. 2003). This remains as one of the most plausible formation mechanisms behinds these features, since nonlinear sausage waves can propagate faster than the sound speed in the magnetically structured solar atmosphere. It is generally assumed that fast MHD pulses are resulting from local magnetic flux emergence and reconnection at the photospheric roots of the magnetic fields.

The bi-directional and cascading nature of the bullets is a very essential new finding of this paper, which suggests that the roots of the involved magnetic field structures are occasionally located sufficiently close such that several bullets may be triggered by the same disturbance. Bi-directional jets are commonly associated with magnetic reconnection events (Innes et al. 1997). Bullets can indeed exhibit bi-directionality, 
but do not behave as jet-like events and an absence of a Doppler velocity component in the $\mathrm{H} \alpha$ line scans of bullets is indicative that the internally heated mass flow argument is not well supported within the best available observations. The propagating MHD pulse model should best describes the overall multithermal structure, evolution, and observational properties of bullets.

The authors are most grateful to the staff of the SST for their invaluable support with the observations. The Swedish 1-m Solar Telescope is operated on the island of La Palma by the Institute for Solar Physics of the Royal Swedish Academy of Sciences in the Spanish Observatorio del Roque de los Muchachos of the Instituto de Astrofísica de Canarias. ES is a Government of Ireland Post-doctoral Research Fellow supported by the Irish Research Council. E.S. thanks Chris Smith for computer access in the Sch. of Physics TCD. The authors wish to acknowledge the DJEI/DES/SFI/HEA Irish Centre for High-End Computing (ICHEC) for the provision of computational facilities (in particular, FIONN cluster) and support. We thank Gregal Vissers for helping with CRISPEX software during the data analyses.

\section{REFERENCES}

Ahmad, I. A., \& Withbroe, G. L. 1977, SoPh, 53, 397

Alexander, C. E., Walsh, R. W., Régnier, S., et al. 2013, ApJL, 775, L32

Antolin, P., \& Rouppe van der Voort, L. 2012, ApJ, 745, 152

Archontis, V., Galsgaard, K., Moreno-Insertis, F., \& Hood, A. W. 2006, ApJL, 645, L161

Archontis, V., \& Hansteen, V. 2014, ApJL, 788, L2

Aschwanden, M. J., \& Boerner, P. 2011, ApJ, 732, 81

Ballai, I., Thelen, J. C., \& Roberts, B. 2003, A\&A, 404, 701

Chiuderi, C., \& Chiuderi Drago, F. 1991, SoPh, 132, 81

Cirtain, J. W., Golub, L., Winebarger, A. R., et al. 2013, Natur, 493, 501

de la Cruz Rodríguez, J., Löfdahl, M. G., Sütterlin, P., Hillberg, T., \& Rouppe van der Voort, L. 2015, A\&A, 573, A40
Engvold, O. 1976, SoPh, 49, 283

Froment, C., Solomon, J., Buchlin, E., et al. 2014, in COSPAR Meeting 40 , 40th COSPAR Scientific Assembly, 903

Hall, L. A., \& Hinteregger, H. E. 1970, JGR, 75, 6959

Heinzel, P., \& Anzer, U. 2001, A\&A, 375, 1082

Heinzel, P., Schmieder, B., Vial, J.-C., \& Kotrč, P. 2001, A\&A, 370, 281

Innes, D. E., Inhester, B., Axford, W. I., \& Wilhelm, K. 1997, Natur, 386, 811

Klimchuk, J. A. 2006, SoPh, 234, 41

Labrosse, N., \& Gouttebroze, P. 2001, A\&A, 380, 323

Labrosse, N., Heinzel, P., Vial, J.-C., et al. 2010, SSRv, 151, 243

Lean, J. 1991, RvGeo, 29, 505

Leenaarts, J., Carlsson, M., \& Rouppe van der Voort, L. 2012, ApJ, 749,136

Lemen, J. R., Title, A. M., Akin, D. J., et al. 2012, SoPh, 275, 17

Lin, Y., Engvold, O., \& Rouppe van der Voort, L. H. M. 2012, ApJ, 747, 129

Morton, R. J., Erdélyi, R., Jess, D. B., \& Mathioudakis, M. 2011, ApJL, 729, L18

Olluri, K., Gudiksen, B. V., \& Hansteen, V. H. 2013, AJ, 145, 72

Roberts, B., \& Mangeney, A. 1982, MNRAS, 198, 7P

Scharmer, G. B. 2006, A\&A, 447, 1111

Scharmer, G. B., Bjelksjo, K., Korhonen, T. K., Lindberg, B., \& Petterson, B. 2003, Proc. SPIE, 4853, 341

Scharmer, G. B., Narayan, G., Hillberg, T., et al. 2008, ApJL, 689, L69

Scullion, E., Rouppe van der Voort, L., Wedemeyer, S., \& Antolin, P. 2014 ApJ, 797, 36

Testa, P., De Pontieu, B., Allred, J., et al. 2014, Sci, 346, B315

Tsuneta, S. 1997, ApJ, 483, 507

van Noort, M., Rouppe van der Voort, L., \& Löfdahl, M. G. 2005, SoPh, 228, 191

Viall, N. M., \& Klimchuk, J. A. 2012, ApJ, 753, 35

Vissers, G., \& Rouppe van der Voort, L. 2012, ApJ, 750, 22

Warren, H. P., Winebarger, A. R., \& Hamilton, P. S. 2002, ApJL, 579, L41

Warren, H. P., Winebarger, A. R., \& Mariska, J. T. 2003, ApJ, 593, 1174

Winebarger, A. R., \& Warren, H. P. 2005, ApJ, 626, 543

Woods, T. 2002, in ESA Special Publication 508, From Solar Min to Max: Half a Solar Cycle with SOHO, ed. A. Wilson (Noordwijk: ESA), 165

Zacharias, P., Peter, H., \& Bingert, S. 2011, A\&A, 532, A112

Zaqarashvili, T. V., Kukhianidze, V., \& Khodachenko, M. L. 2010, MNRAS, 404, L74 